\title{
Comparisons Between NO PLIF Imaging and CFD Simulations of Mixing Flowfields for High-Speed Fuel Injectors.
}

\author{
Tomasz G. Drozda, ${ }^{\dagger}$ Karen F. Cabell,$^{\dagger}$ Austin R. Ziltz, ${ }^{\ddagger}$ Neil E. Hass, ${ }^{\dagger}$ \\ Hypersonic Airbreathing Propulsion Branch, NASA Langley Research Center, Hampton, VA, 23681 \\ Jennifer A. Inman, ${ }^{\dagger}$ Ross A. Burns,${ }^{\S}$ Brett F. Bathel, ${ }^{\dagger}$ Paul M. Danehy, ${ }^{\S}$ \\ Advanced Measurement and Data Systems Branch, NASA Langley Research Center, Hampton, VA, 23681 \\ Yasin M. Abul-Huda, " Mirko Gamba** \\ Department of Aerospace Engineering, University of Michigan, Ann Arbor, MI, 48109
}

\begin{abstract}
The current work compares experimentally and computationally obtained nitric oxide (NO) planar laser-induced fluorescence (PLIF) images of the mixing flowfields for three types of highspeed fuel injectors: a strut, a ramp, and a rectangular flushwall. These injection devices, which exhibited promising mixing performance at lower flight Mach numbers, are currently being studied as a part of the Enhanced Injection and Mixing Project (EIMP) at the NASA Langley Research Center. The EIMP aims to investigate scramjet fuel injection and mixing physics, and improve the understanding of underlying physical processes relevant to flight Mach numbers greater than eight. In the experiments, conducted in the NASA Langley Arc-Heated Scramjet Test Facility (AHSTF), the injectors are placed downstream of a Mach 6 facility nozzle, which simulates the high Mach number air flow at the entrance of a scramjet combustor. Helium is used as an inert substitute for hydrogen fuel. The PLIF is obtained by using a tunable laser to excite the NO, which is present in the AHSTF air as a direct result of arc-heating. Consequently, the absence of signal is an indication of pure helium (fuel). The PLIF images computed from the computational fluid dynamics (CFD) simulations are obtained by combining a fluorescence model for NO with the Reynolds-Averaged Simulation results carried out using the VULCAN-CFD solver to obtain a computational equivalent of the experimentally measured PLIF signal. The measured NO PLIF signal is mainly a function of NO concentration allowing for semiquantitative comparisons between the CFD and the experiments. The PLIF signal intensity is also sensitive to pressure and temperature variations in the flow, allowing additional flow features to be identified and compared with the CFD. Good agreement between the PLIF and the CFD results provides increased confidence in the CFD simulations for investigations of injector performance.
\end{abstract}

\section{Introduction}

C LIGHT demonstrations of hypersonic air-breathing vehicles $^{1,2}$ prove their increasing promise for military (rapid

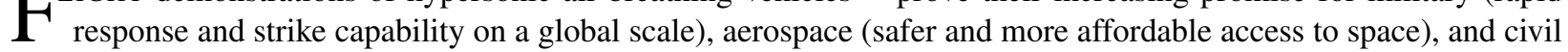
aviation (hypersonic point-to-point transport) applications. However, designing supersonic combustion ramjet (scramjet) engines capable of robust air-breathing operation, characterized by rapid fuel-air mixing and short combustion times while ensuring flame stability and high combustion efficiency over a wide range of speeds, has proven difficult. Attempts at improving fuel injection to enhance fuel-air mixing while simultaneously reducing losses have received

\footnotetext{
${ }^{\dagger}$ Research Aerospace Engineer, AIAA Senior Member.

${ }^{\ddagger}$ Research Engineer, ACEnT Labs

$\S$ Research Engineer, National Institute of Aerospace, AIAA Senior Member.

TNASA Langley Senior Technologist, AIAA Associate Fellow.

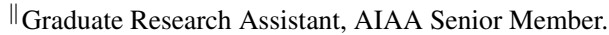

** Assistant Professor, AIAA Senior Member.
} 
a great deal of attention over the years, as summarized by Lee et al. ${ }^{3}$ Although some total pressure loss is thermodynamically unavoidable and occurs due to the desired effect of molecular mixing of the fuel and air, any losses beyond this minimum required amount reduce the thrust potential of the engine.

The Enhanced Injection and Mixing Project (EIMP), being executed at the NASA Langley Research Center, represents an effort to achieve more rapid mixing at high speeds. ${ }^{4}$ The EIMP aims to investigate scramjet fuel injection and mixing physics, improve the understanding of underlying physical processes, and develop enhancement strategies relevant to flight Mach numbers greater than eight. The experiments, which are underway in the Arc-Heated Scramjet Test Facility (AHSTF), utilize a "semi-freejet," subcomponent-level approach with a Mach 6 facility nozzle to produce a flow field that simulates a nondistorted combustor entrance flow of a scramjet engine. The fuel injectors tested to date consist of a fuel placement device, a strut; a fluidic vortical mixer, a ramp; and a high aspect ratio rectangular flushwall injector. These devices accomplish the necessary task of distributing and mixing fuel into the supersonic crossflow, albeit via different strategies. These devices were previously studied at lower flight Mach numbers where they exhibited promising performance in terms of mixing efficiency and total pressure recovery. ${ }^{5,6}$ Computational fluid dynamics (CFD) simulations, and the corresponding comparative analysis of these injectors under the current conditions of interest, were also previously investigated. ${ }^{7}$

The EIMP experiments include quantitative in-stream measurements as well as flow visualization of the mixing flowfield via the nitric oxide (NO) planar (P) laser-induced fluorescence (LIF) technique, or NO PLIF. The present application takes advantage of the fact that NO naturally exists in low concentrations in the facility air ${ }^{8}$ as a result of the electric-arc heating process. The NO acts as an in situ flow tracer that can be imaged using PLIF. NO PLIF uses an ultraviolet (UV) laser sheet to illuminate a planar slice in the flow. The UV light excites fluorescence from the NO molecules, which is detected by a digital camera. The PLIF technique has been employed in numerous supersonic fuel-air mixing studies ${ }^{9-17}$. The present application follows closely the method of Fox et al., ${ }^{13}$ where NO is in the air stream instead of the fuel (helium) stream, thus the absence of signal is an indication of pure fuel and the intensity of the fluorescence is proportional to the concentration of NO.

Prior to conducting mixing tests, earlier experiments were conducted in the AHSTF with the laser sheet configured to interrogate the flow at the exit of the Mach 6 facility nozzle. ${ }^{18}$ These tests confirmed that NO exists in sufficient concentration (i.e., to produce enough fluorescence to detect) and sufficient spatial uniformity for images obtained from the mixing flowfield to be interpreted properly (i.e., concentration of NO proportional to concentration of air). The initial set of mixing tests of the injectors described above has been conducted using only NO PLIF imaging and no intrusive measurements. Both streamwise and cross-stream PLIF images have been obtained. Because the instantaneous concentration of NO is not precisely known or controlled, this experimental approach presents a unique set of challenges from those where a known amount of NO is seeded into the flow. Furthermore, the measured NO PLIF signal is also sensitive to pressure and temperature variations, both of which can vary significantly in a compressible flow with shock and expansion features. Nevertheless, the current approach is similar to previous work of Fox et al., ${ }^{13}$ and Gaston et al. ${ }^{14}$

The PLIF images computed from the CFD are referred to as the computational flow imaging (CFI), and are obtained by combining a fluorescence model for $\mathrm{NO}^{19}$ with the Reynolds-Averaged Simulation results carried out using the VULCAN-CFD solver. ${ }^{20}$ The resulting images are equivalent to the experimentally measured PLIF signal, and are proportional to the NO concentration, and simultaneously exhibit the correct amount of sensitivity to both temperature and pressure. However, due to the uncertainties associated with the instantaneous amount of background NO, the absorption model (or lack thereof), PLIF signal postprocessing, and the PLIF signal modeling itself, the comparisons between CFI and PLIF are only semiquantitative. Nevertheless, good agreement between the CFI and PLIF provides increased confidence in the CFD simulations and the corresponding CFD analysis that investigates the injector performance (e.g., Drozda et al. ${ }^{7}$ ).

\section{Mixing Experiments}

EIMP experiments entail testing various fuel injection devices mounted on an open flat plate located downstream of a Mach 6 facility nozzle, which simulates the combustor entrance of a flight vehicle traveling at a Mach number of about 14 to 16 . The open flat plate is used because it facilitates optical access to the test region. Figure 1 shows the cross section of the experimental apparatus mounted in the $4 \mathrm{ft}$ diameter test cabin. Noted on the figure is the flat plate test bed platform with its leading edge positioned 2.5 inches below the top wall of the Mach 6 facility nozzle. The flow is from left to right. The flat plate is approximately 29 in long tip-to-tail and 32 in wide, and features an interface for mounting interchangeable injector blocks. The trailing edge of the injector block is located 8.87 in downstream of 


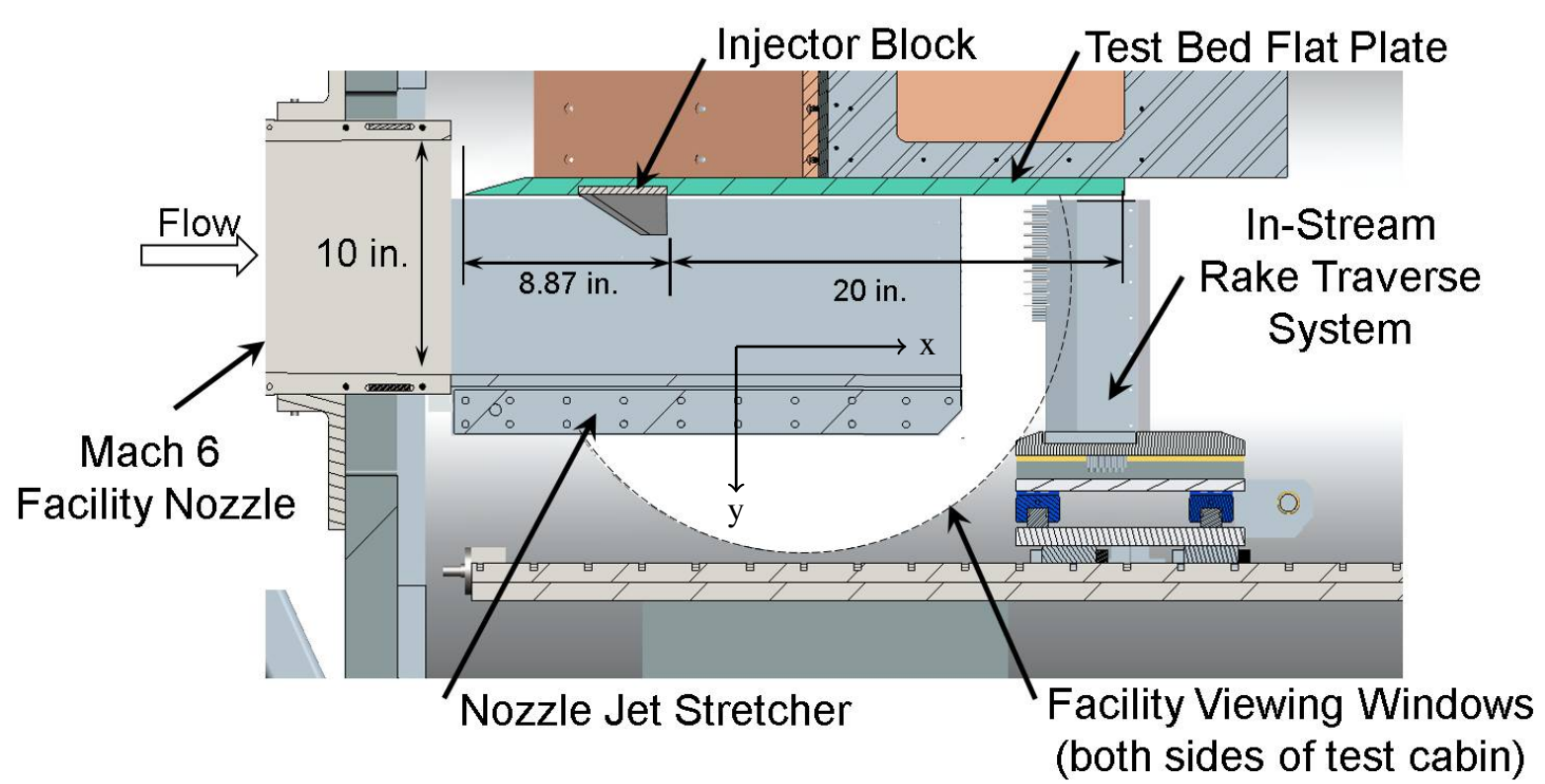

Figure 1. Cross section of the experimental cabin with the facility nozzle, test bed plate, jet-stretchers, instrumentation shroud, and the in-stream rake probe indicated. The figure shows the configuration as installed in the wind tunnel. All CFD simulations were performed with the positive y-direction in the plate-normal direction, which is pointing toward the ground.

the test bed plate leading edge. The injector blocks, in general, can accommodate a spanwise row of several injectors. The facility air flows over the injector bodies and mixes with the fuel simulant (helium) downstream of the injection plane. Also noted in Fig. 1 are the in-stream rake traverse system (used for pitot pressure, total temperature, and gas sampling probes), the jet stretchers, and the instrumentation shroud located on the upper side of the flat plate. In the current experiments, neither the in-stream rake traverse nor the jet-stretchers are installed. The figure also shows the orientation of the experimental apparatus as is installed in the AHSTF test cabin. In all subsequent CFD simulations the positive y-direction is oriented in the plate-normal direction, which in this figure is pointing toward the ground. Further details about the experimental setup and EIMP are presented in Cabell et al. ${ }^{4}$ The experiment is intended to provide a test-bed for rapidly testing a variety of different fuel injector devices and injection strategies. Current experiments focus on studying fuel injection and mixing processes in the absence of heat release by utilizing helium as a fuel simulant. Furthermore, these experiments are referred to as "cold flow" because the range of air total temperatures is significantly lower than the relevant flight total temperature. The low end of the range is bounded by the condensation limit for the expanding facility air and the high end by the thermal-structural limits of the uncooled hardware. The former and latter correspond to 728 and $978 \mathrm{~K}$, respectively. However, the Reynolds numbers are comparable between the ground experiments and flight. Only the upper limit of the total temperature is included in the current study of the EIMP case because previous work ${ }^{21}$ has shown that the results obtained for the lower limit are similar.

\section{Nominal Flow Conditions and Injector Geometries}

The nominal facility air conditions correspond to a total pressure and total temperature of $4.31 \mathrm{MPa}$ (625 psi) and $978 \mathrm{~K}\left(1760^{\circ} \mathrm{R}\right)$, respectively, expanded to a Mach number of $6.4 .^{22}$ A nonreacting, thermally perfect mixture of $21 \%$ oxygen $\left(\mathrm{O}_{2}\right), 78 \%$ nitrogen $\left(\mathrm{N}_{2}\right)$, and $1 \%$ nitric oxide $(\mathrm{NO})$ by volume was assumed for the air. ${ }^{8}$ The fuel mass flow rate of helium for each injector was computed by assuming an equivalence ratio of one over the "intended" fueling area as if it were fueled with hydrogen. The intended fueling areas for the strut and ramp are portions of the cross-sectional area of the complete combustor flowpath that are intended to be fueled by a single injector with the equivalence ratio of one. For the strut and ramp injectors, the intended fueling areas were obtained from Baurle et al., ${ }^{5}$ who considered them in a realistic scramjet combustor configuration. The values of several flow parameters are shown in Table 1. 
Table 1. Nominal global parameters of interest for the strut, ramp, and flushwall injector configurations. The last set of rows contain ratios of interest between the fuel (helium) and air streams, where the subscripts $f$ and $a$ denote fuel and air streams, respectively.

\begin{tabular}{|c|cccc|}
\hline Property & Air $^{\dagger}$ & Strut & Ramp & Flushwall \\
\hline Mach & 6.36 & 2.98 & 2.96 & 2.98 \\
$P 0(\mathrm{MPa})$ & 4.309 & 0.224 & 0.0882 & 0.424 \\
$T 0(\mathrm{~K})$ & 977.8 & 293.15 & 293.15 & 293.15 \\
$P(\mathrm{kPa})$ & 1.808 & 7.205 & 2.911 & 13.642 \\
$T(\mathrm{~K})$ & 112.4 & 74.14 & 74.91 & 74.14 \\
$\mathrm{u}(\mathrm{m} / \mathrm{s})$ & 1353 & 1508 & 1505.6 & 1508.2 \\
$R e^{\prime}(1 / \mathrm{in}) \times 10 \mathrm{e} 3$ & 259.4 & 358.4 & 143.1 & 678.6 \\
\hline$\dot{\mathrm{m}}_{\mathrm{a}}(\mathrm{kg} / \mathrm{s}) \times 10 \mathrm{e}-3$ & & 98.76 & 65.84 & 187.00 \\
$\mathrm{~m}_{\mathrm{f}}(\mathrm{kg} / \mathrm{s}) \times 10 \mathrm{e}-3$ & & 2.884 & 1.922 & 5.460 \\
\hline$\Delta \mathrm{U}^{\ddagger}$ & & 0.054 & 0.054 & 0.054 \\
$\mathrm{M}_{\mathrm{c}}{ }^{\S}$ & & 0.22 & 0.21 & 0.22 \\
$\rho_{\mathrm{f}} / \rho_{\mathrm{a}}$ & & 0.84 & 0.33 & 1.58 \\
$\mathrm{p}_{\mathrm{f}} / \mathrm{pa}_{\mathrm{a}}$ & & 3.98 & 1.61 & 7.54 \\
$J^{\uparrow}$ & & 1.04 & 0.42 & 1.97 \\
\hline
\end{tabular}

$\dagger 21 \% \mathrm{O}_{2}, 78 \% \mathrm{~N}_{2}, 1 \% \mathrm{NO}$

$\ddagger$ Velocity difference parameter, $\Delta U=\left(u_{f}-u_{a}\right) /\left(u_{f}+u_{a}\right)$

${ }^{\S}$ Convective Mach number, $M_{c}=\left|u_{f}-u_{a}\right| /\left(c_{f}+c_{a}\right), c$ denotes the speed of sound.

IDynamic pressure ratio, $J=\left(\rho_{f} u_{f}^{2}\right) /\left(\rho_{a} u_{a}^{2}\right)$.

Both the properties of air and fuel are presented. The subscripts $0, f$, and $a$ denote total conditions, fuel, and air flow streams, respectively. It should be noted that these values correspond to the nominal conditions used in, or obtained from, the CFD simulations. The quantities that were measured experimentally varied slightly from run-to-run from the nominal values. The following nondimensional quantities are also shown: the unit Reynolds number per inch for the air and fuel streams; velocity difference parameter, $\Delta \mathrm{U}$; the convective Mach number, $\mathrm{M}_{\mathrm{c}}$; the ratios of the density, $\rho_{\mathrm{f}} / \rho_{\mathrm{a}}$, and static pressure, $\mathrm{p}_{\mathrm{f}} / \mathrm{p}_{\mathrm{a}}$; and dynamic pressure, $J$, between the fuel and air stream. All values are computed based on the combustor entrance flow conditions for the air and the expanded flow conditions at the exit of the injector ports for the fuel. These nondimensional quantities have been found to be relevant to the injection and mixing processes in canonical problems. ${ }^{23-25}$

As stated earlier, the three types of injectors investigated in the current study are a strut, a ramp, and a flushwall injector. The same strut and ramp have been previously studied by Baurle et al. ${ }^{5}$ under "cold" flow conditions at a combustor entrance Mach number of 4.5. However, unlike the experiments and simulations discussed in Baurle et al., ${ }^{5}$ which configured the injectors in a closed duct and interdigitated fashion, the current work includes a row of injectors on an open flat plate. The flushwall injector is a rectangular, high-aspect ratio design based on the optimization work of Ogawa ${ }^{6}$ at Mach 5.7 flow conditions. Although the computational simulations included a row of flushwall injectors, only a single injector was able to be accommodated in the current experiments.

The first injector is a slender swept strut protruding into the flow. Strut injectors have several advantages in hypervelocity flow applications. First, they can be designed to place the fuel where it is needed, thereby alleviating the need to consider fuel penetration issues and focusing only on the injector spacing. Second, the injector ports on a strut are typically aligned parallel to the flow, which allows the injected fuel streams to augment the thrust of the engine. The potential downsides of a strut injector are the structural integrity and cooling requirements needed for its slender body, the drag (both viscous and pressure) that it induces on the flow by the obstruction it generates, and the total pressure loss incurred by the oblique shocks that emanate from its body. Views and dimensional details of the strut, are shown in Fig. 2. The $\mathrm{x}, \mathrm{y}$, and $\mathrm{z}$ denote the streamwise or downstream, vertical or wall normal, and cross-stream directions, respectively. In the current simulations and experiments, the struts are placed 0.9 inches apart. Each injector port has a throat diameter of 0.083 inches followed by a conical expansion with a half-angle of 6 degrees that is designed to expand helium to a Mach number of about 3.

The second injector is an unswept ramp. Compared to the strut, the ramp injector does not protrude as far into the flow. The ramp injector generates a counter-rotating vortex pair (CVP) on each side of the ramp as the flow passes over the ramp and through the gaps between the ramps, which convects the injected fuel upward, toward the core of 


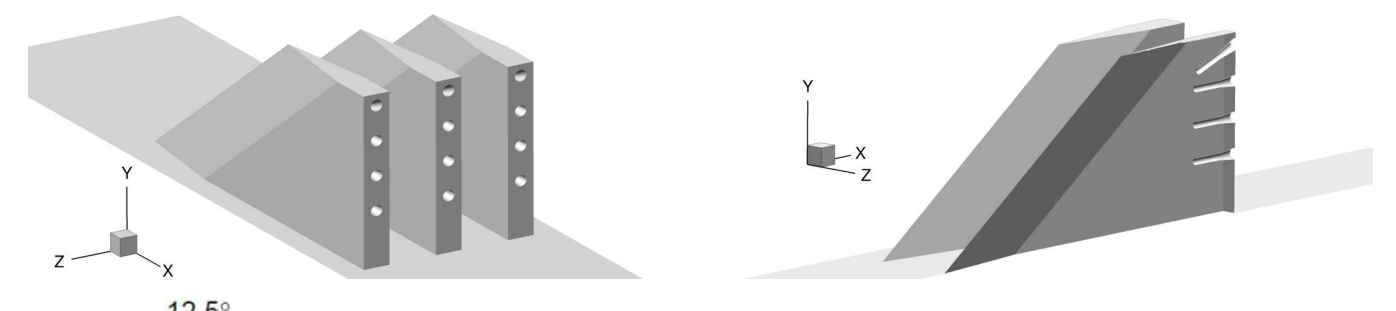

$12.5^{\circ}$

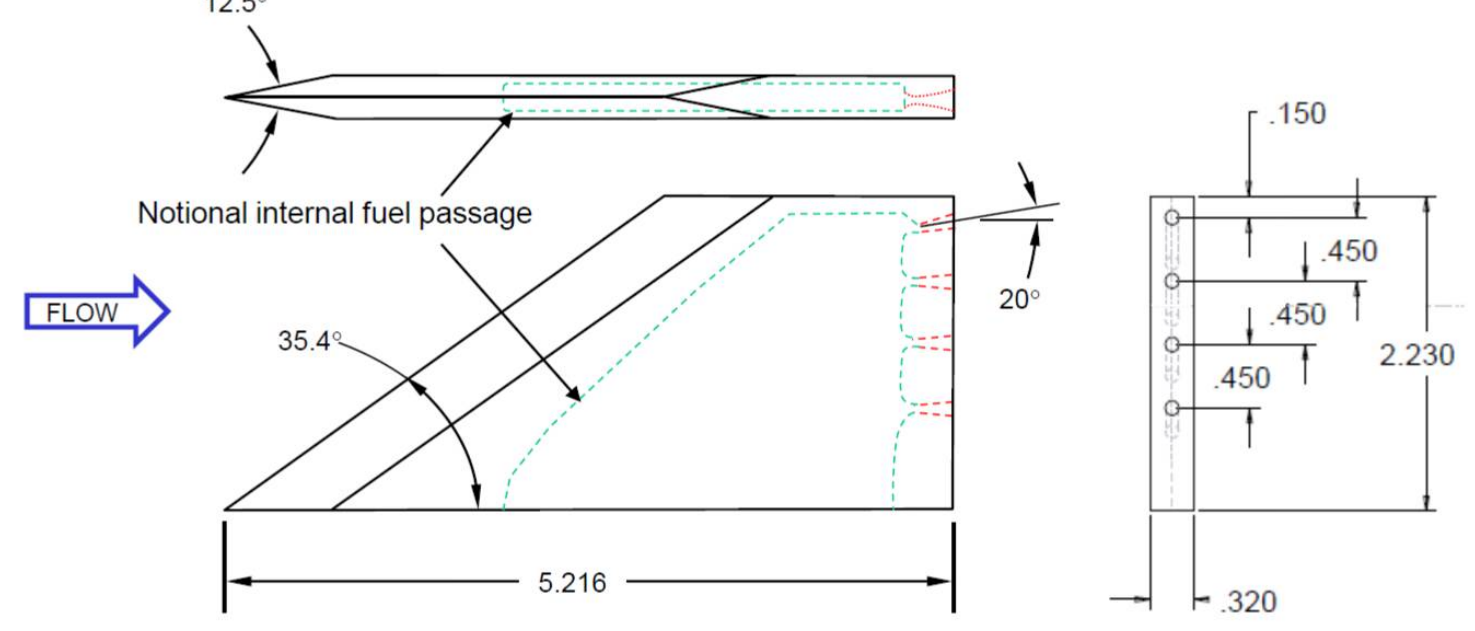

Figure 2. Isometric views and dimensional details of the baseline strut injector. Linear dimensions are in inches.

the flow. In addition to inducing the upward entrainment, the CVP stretches the fuel-air interface thereby increasing the surface area over which the turbulence and the molecular viscosity can act to mix the fuel and air. Because the strength of the CVP depends on both the geometry of the ramp, and the incoming air flow conditions, designing an injector that robustly fills its intended fueling area across a range of flight conditions is more challenging for a ramp than a strut. As with the strut, the ports of the ramp injector are also nearly aligned parallel to the flow and allow for injected fuel streams to augment the thrust of the engine. However, the ports are angled slightly upward and to the side to aid in directing the fuel streams toward the CVP. The ramp injector also generates an oblique shock due to its inclined surface. Views of the ramp, and its dimensional details are shown in Fig. 3. In the current simulations, the adjacent ramps are placed 1.2 inches apart. This spacing is also the same as that found between the interdigitated ramp configuration of Baurle et al. ${ }^{5}$ Each injector port has a throat diameter of 0.108 inches followed by a conical expansion with a half-angle of 10 degrees that is designed to expand helium to a Mach number of about 3.

The third injector is a high-aspect-ratio rectangular flushwall injector. Unlike the strut and ramp, the flushwall injector introduces no physical blockage into the flow. Instead, a number of flow features form around the injection site that interact to produce a similar effect. These features are shown schematically for a generic round flushwall injector in Fig. 4. The bow shock that forms upstream of the injection plume creates both total pressure losses and aerodynamic blockage by forcing the air stream to flow around the fuel plume. As is the case for the ramp injector, the fuel plume entering the high-speed cross flow generates a CVP, which becomes the main mechanism for stirring the fuel into the air. However, unlike a fuel placement device, such as a strut, the extent to which the fuel penetrates into the air flow is governed by fluidic considerations. ${ }^{3,27-29}$ The jet penetration has been shown to be primarily proportional to the dynamic pressure (or momentum flux) ratio, $J$, between main air and the fuel jet, and is further enhanced by matching the static pressure at the exit of the fuel injector to the effective static pressure, that is, the static pressure of the air just upstream of the fuel plume and downstream of the bow shock. Penetration also increases with the increasing thickness of the approach boundary layer ${ }^{30}$ due to aerodynamic "shielding."

The flushwall injector exit geometry is based on the multiobjective optimization work of Ogawa ${ }^{6}$ whose approach using a genetic algorithm revealed four families of high performing flushwall injectors. The injector port chosen for the current work has a constant width, rectangular cross-section, with an aspect ratio of 8 at the injector exit plane, and the longer dimension aligned in the streamwise direction. The injector is also inclined at 30 degrees to the wall. 

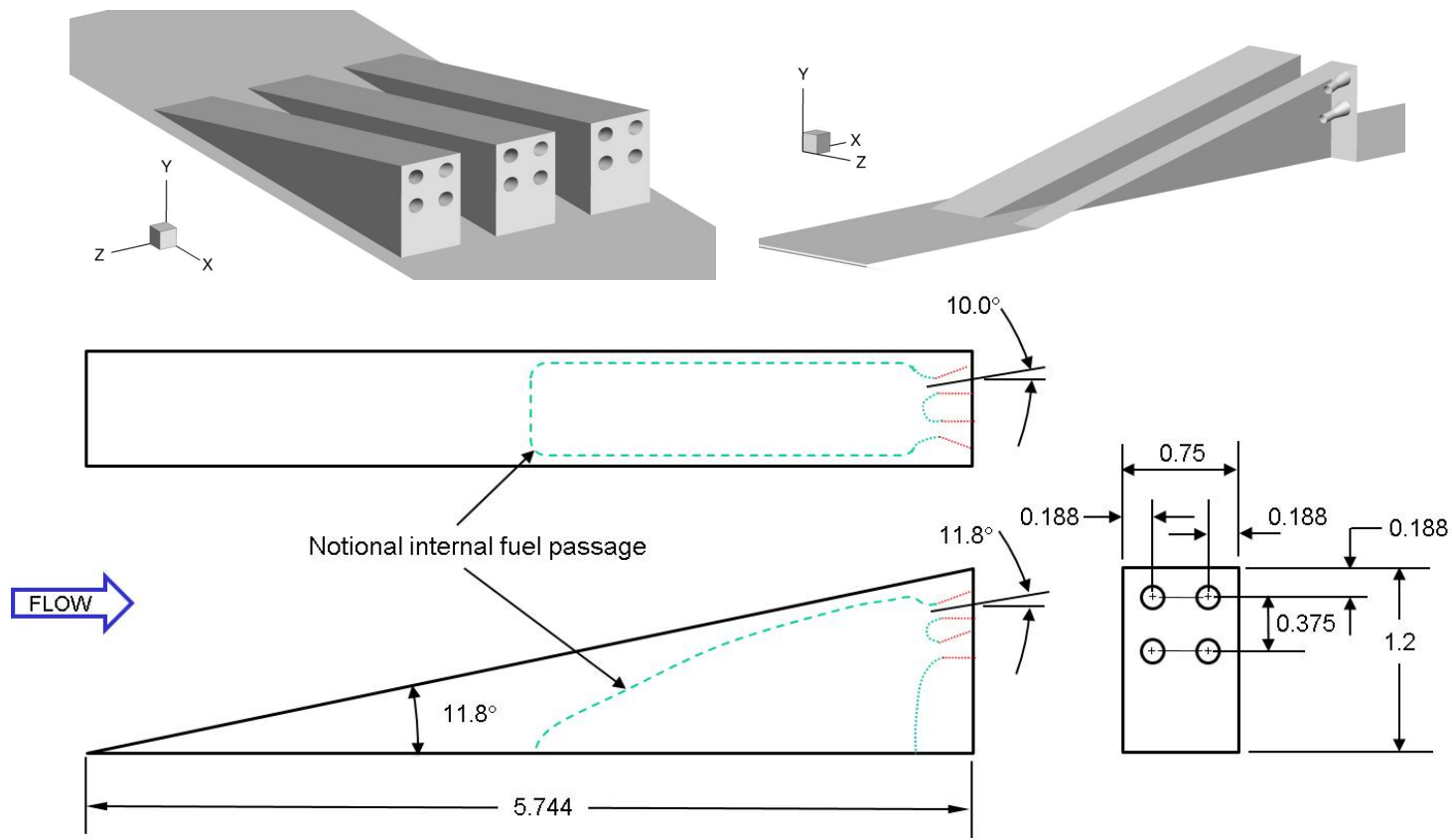

Figure 3. Isometric views and dimensional details of the baseline ramp injector. Dimensions are in inches.
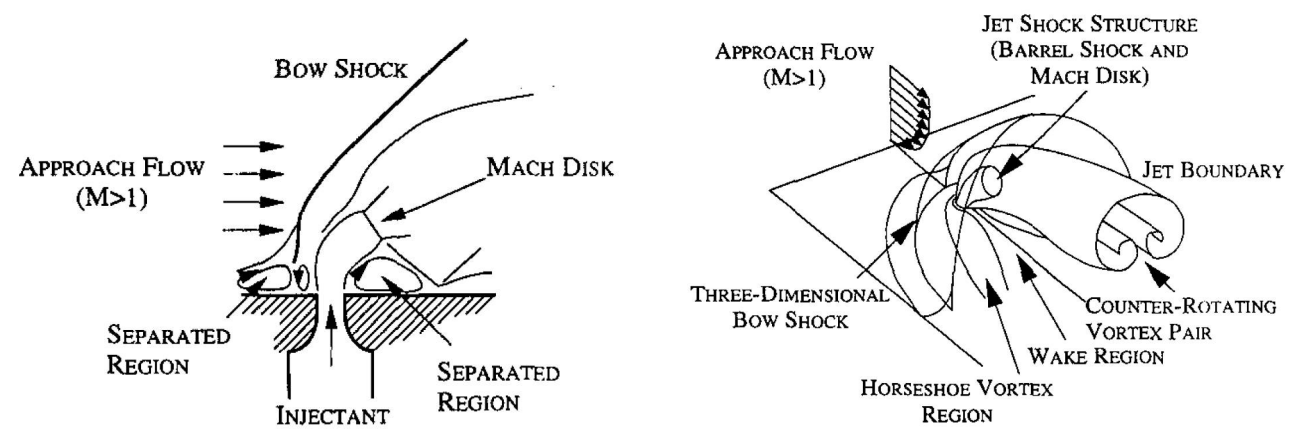

Figure 4. Side and isometric views of the flow features that form around a generic flushwall injector during transverse injection of fuel into the supersonic cross-stream. From Gruber et al. ${ }^{26}$

Several views, and dimensional details of the flushwall injector are shown in Fig. 5. The injector has been further designed to qualitatively match the geometrical features of the fuel ports of the strut injector. As such, the flushwall injector contains an expansion section with a 6 degrees half-angle, and an expansion area ratio matching that of the strut conical fuel port. The area at the end of the expansion section, but before the 30-degree rotation, has been adjusted to match the total exit area of the 4 fuel ports of the strut. The width of the injector is 0.1392 inches with a throat height of 0.1541 inches. In the CFD simulations, the adjacent flushwall injectors are placed 1.704 inches apart, however, the experimentally tested flushwall injector block contains only a single injector. The spacing corresponds to about 6 times the diameter of a circular injector with an equivalent area, and about 1.5 times the length of the current injector, allowing for a sufficient air-gap between the adjacent injectors even after the expected axis-switching of the fuel plume. ${ }^{6}$ 


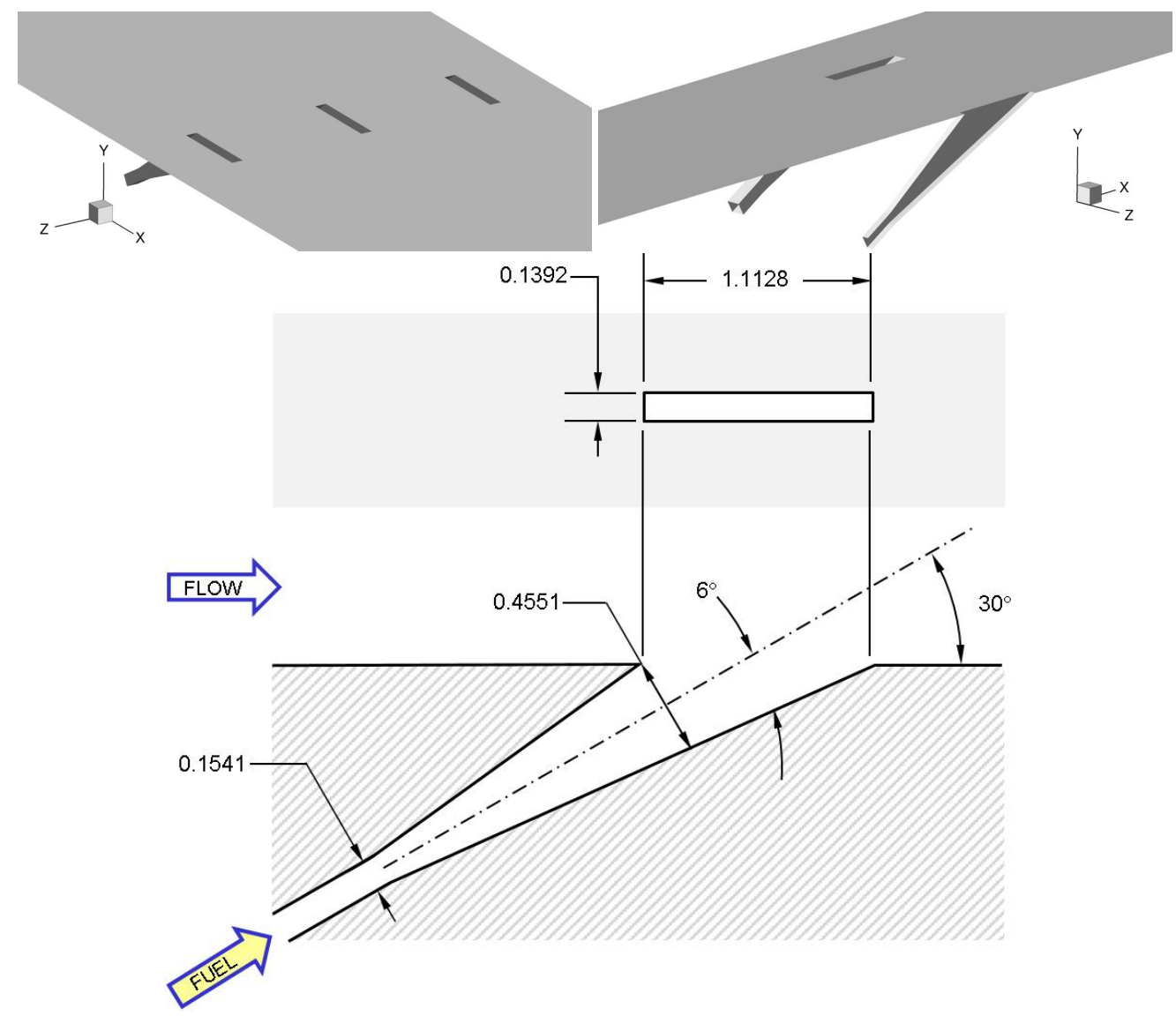

Figure 5. Isometric views and dimensional details of the baseline flushwall injector.

\section{Computational Modeling}

The numerical simulations were performed using the Viscous Upwind aLgorithm for Complex flow ANalysis (VULCAN-CFD) code. ${ }^{20}$ VULCAN-CFD is a multiblock, structured-grid, cell-centered, finite-volume solver widely used for all-speed flow simulations. For this work, Reynolds-averaged simulations (RAS) were performed. The advective terms were computed using a Monotonic Upstream-Centered Scheme for Conservation Laws (MUSCL) scheme $^{31}$ with the Low-Dissipation Flux-Split Scheme (LDFSS) of Edwards ${ }^{32}$ The thermodynamic properties of the mixture components were computed using curve fits of McBride et al. ${ }^{33}$ The governing equations were integrated using an implicit diagonalized approximate factorization (DAF) method. ${ }^{34}$ The current work used the baseline blended $\mathrm{k}-\omega / \mathrm{k}-\epsilon$ turbulent physics model of Menter. ${ }^{35}$ The Reynolds heat and species mass fluxes were modeled using a gradient diffusion model with turbulent Prandtl and Schmidt numbers of 0.9 and 0.5 , respectively. These values were set based on experience and best practice with similar flows. Wilcox wall matching functions ${ }^{36}$ were also used, however, their implementation in VULCAN-CFD includes a modification that allows the simulations to recover the integrate-to-the-wall behavior as the value of y+ approaches one. All simulations were converged until the total integrated mass flow rate and the total integrated heat flux on the walls remained constant to within six significant digits. This typically occurred when the value of the $\mathrm{L}_{2}$-norm of the steady-state equation-set residual decreased by about 4-5 orders of magnitude. The grid dependence analysis for the numerical simulations of both the strut and ramp injectors were previously assessed by Drozda et al. ${ }^{7}$ The numerical simulations took advantage of the geometric symmetries of the injectors, therefore, only a single injector is simulated with the symmetry boundary conditions used at the midplane between the adjacent injectors. It should also be noted that all of the CFD simulations were performed pretest, and no attempt was made to rerun the simulations to match the experimental flow conditions (e.g., total pressure and temperature, Mach number, and equivalence ratio) exactly. 

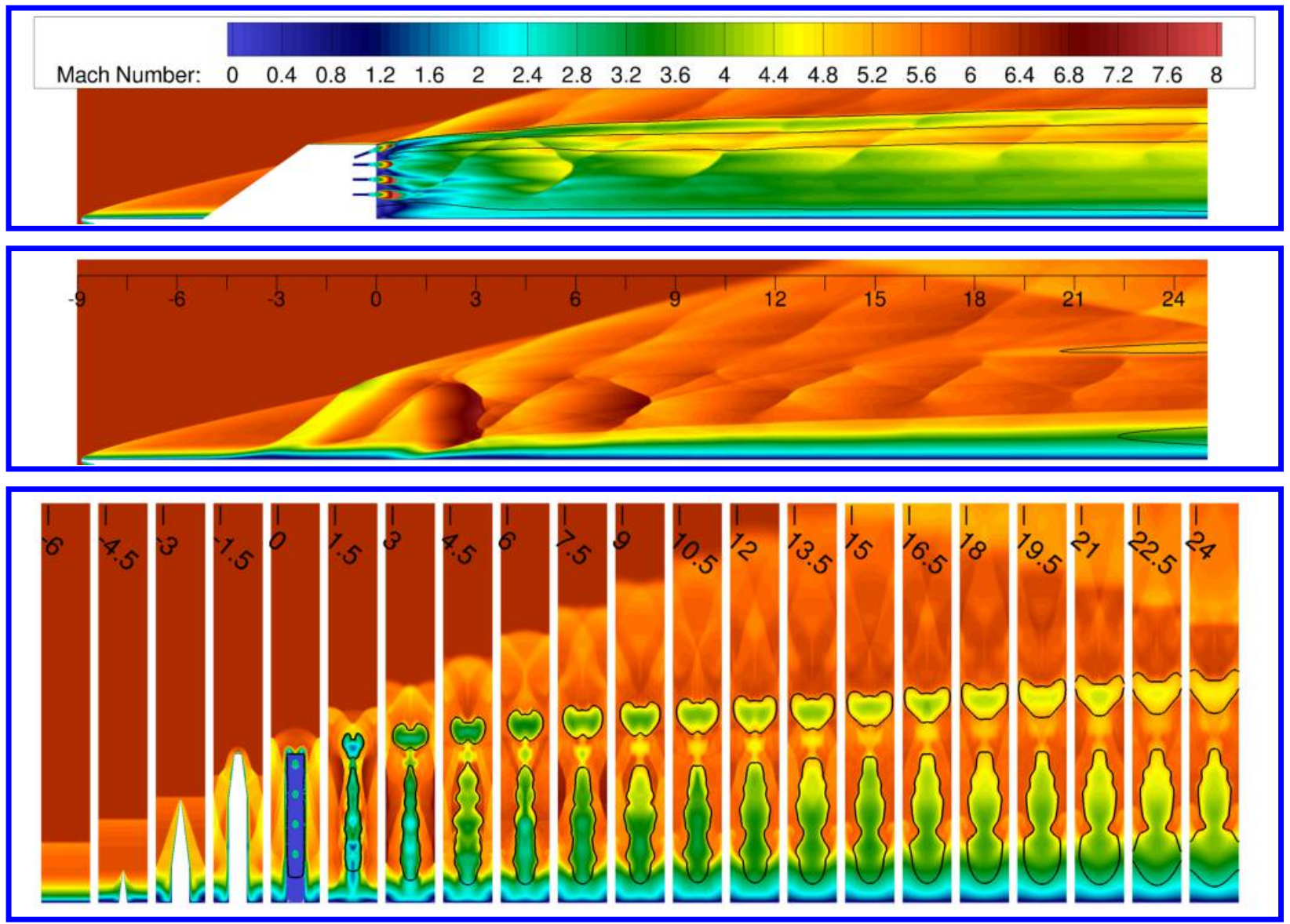

Figure 6. Contours of the Mach number on the z-planes obtained at the centerline, and half-way between the injectors, and x-planes at various downstream locations for the strut injector simulations. Downstream distance is in inches. Black lines denote the stoichiometric value of the fuel mass fraction.

Contour plots of the Mach number in the z-planes obtained through the center of the injector ports and midway between the injectors, and in x-planes at various locations for the strut, ramp, and the flushwall injectors are shown in Figs. 6-8, respectively. The flow is left-to-right. For the x-planes, the aspect ratio is not equal to one because these planes are viewed looking aft-to-fore from an angle of about 30 degrees to the $x$-axis in the xz-plane. The streamwise distance on these figures is in inches. The black isocontour line denotes a helium mass fraction equal to the stoichiometric value for hydrogen (0.0285).

Qualitatively, the flow features for all of the injectors are very similar. Upstream, the leading edge of the flat plate causes a shallow bow shock at about 12.5 degrees to the flat plate, which is slightly larger than the Mach wave angle of about 9 degrees for this Mach number. For the strut injector, shown in Fig. 6, a cross-stream shock wave is generated by the sharp leading edge of the strut injector body (e.g., at -3 inches). The turning half-angle of the strut injector body leading edge is 6.25 degrees (see Fig. 2), resulting in the leading edge shock wave angle of about 17 degrees as measured from the CFD. Because the leading edge of the strut body is swept at 35.4 degrees, the resulting oblique shock wave forms at a somewhat larger angle than that expected for the turning angle of 6.25 degrees. This shock wave propagates in the cross-stream direction and impacts the body of the adjacent injector. This propagation is evident by the change in the value of the Mach number at downstream locations between -3 and 0 inches on the middle plots of each figure. After the reflection from the adjacent injector, the oblique shock waves continue to pass through one another and interact, leading to the complex downstream pattern seen in the figures. As the flow continues past the strut injector body, a counter rotating vortex pair (CVP) forms near the tip of the injector body. The top-most fuel port injects the fuel stream directly into the space between the CVP. The combined effect of the angled injection and the CVP distorts and bifurcates the top-most fuel stream, and separates it from those of the three lower parallel fuel 

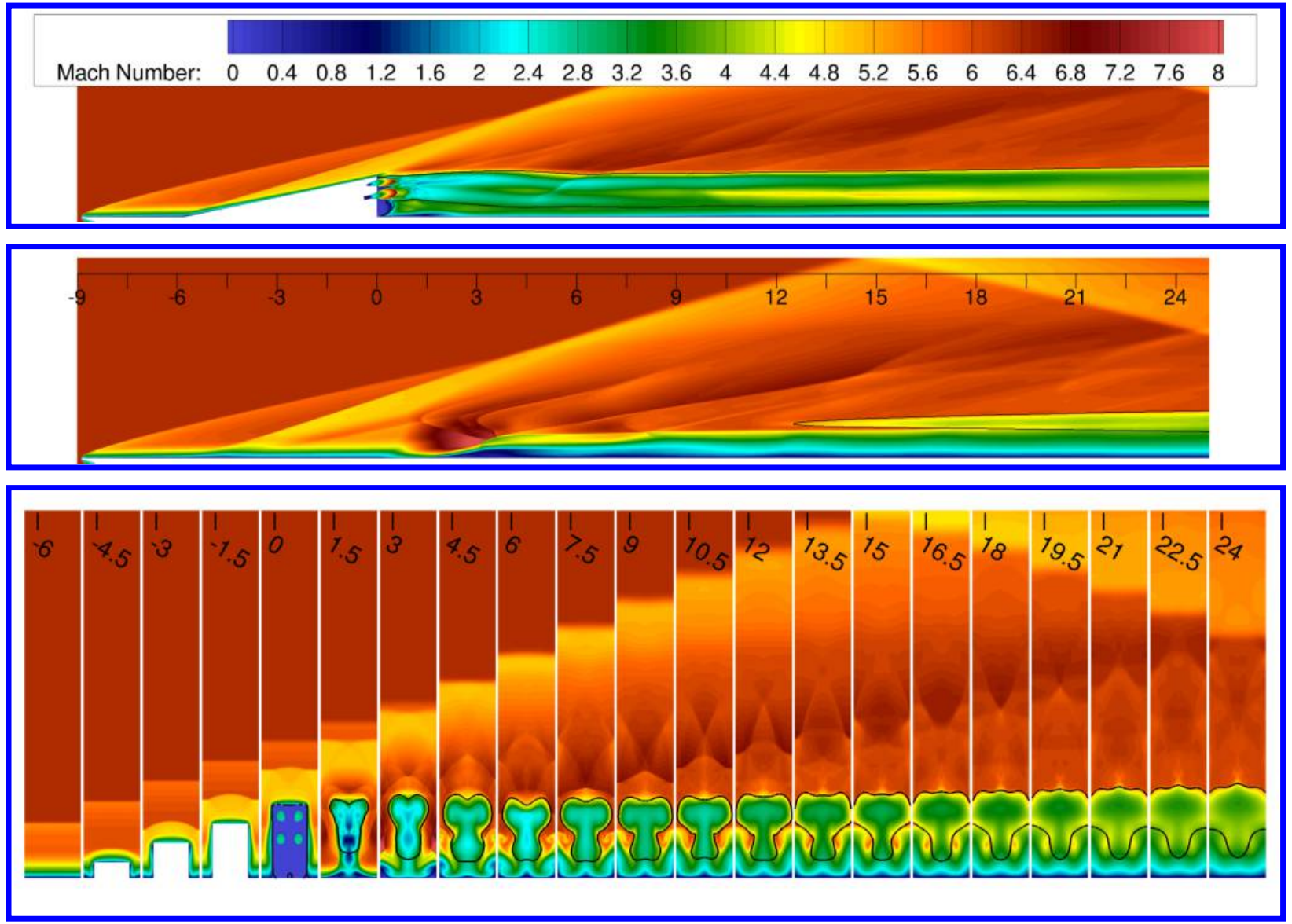

Figure 7. Contours of the Mach number on the z-planes obtained through the center of the injector ports, and half-way between the injectors, and $x$-planes at various downstream locations for the ramp injector simulations. Downstream distance is in inches. Black lines denote the stoichiometric value of the fuel mass fraction.

ports. This effect can be seen by observing the stoichiometric value of the mass fraction as it evolves in the x-planes in Fig. 6.

For the ramp injector, shown in Fig. 7, an oblique shock wave is generated by the inclined ramp surface of the ramp injector body. The turning angle of the ramp surface is 11.8 degrees (see Fig. 3), resulting in the oblique shock wave angle of about 17 degrees at the injector centerline. The value of this angle is slightly reduced from the expected value (of about 19 degrees) for this turning angle because of the interaction of the shock with the approach boundary layer. This oblique shock interacts and coalesces with the same shock wave produced by the adjacent injector bodies. However, unlike the cross-stream shock waves that emanate from the leading edge of the strut, the ramp body oblique shock wave does not interact with the fuel-air mixing plume but instead serves primarily to introduce a pressure difference between the top of the ramp surface and the gap between the adjacent injectors. The pressure is higher on the ramp surface, which creates a driving force for the flow to spill from the ramp surface into the gap between the adjacent injectors. This spillage introduces large counter rotating vortices on either side of the ramp injector body with the size and circulation proportional to the ramp height. ${ }^{37}$ These vortices are large compared to the size of the fuel ports. Therefore, when they begin to interact with the injected fuel streams, they stretch and push the fuel-air interface upward away from the plate boundary layer and into the core of the flow. However, it should be noted that for the current ramp injector, the CVP is constrained by the narrow spacing between the adjacent injectors, which limits the size and strength of the CVP, and therefore, the upward movement of the fuel plume. This movement of the fuel into the core flow is, however, aided by the fact that all the ramp fuel ports are angled at 11.8 degrees up and the top set is angled at 10 degrees outward toward the gap between the adjacent injectors. Nevertheless, the combined effect of the 

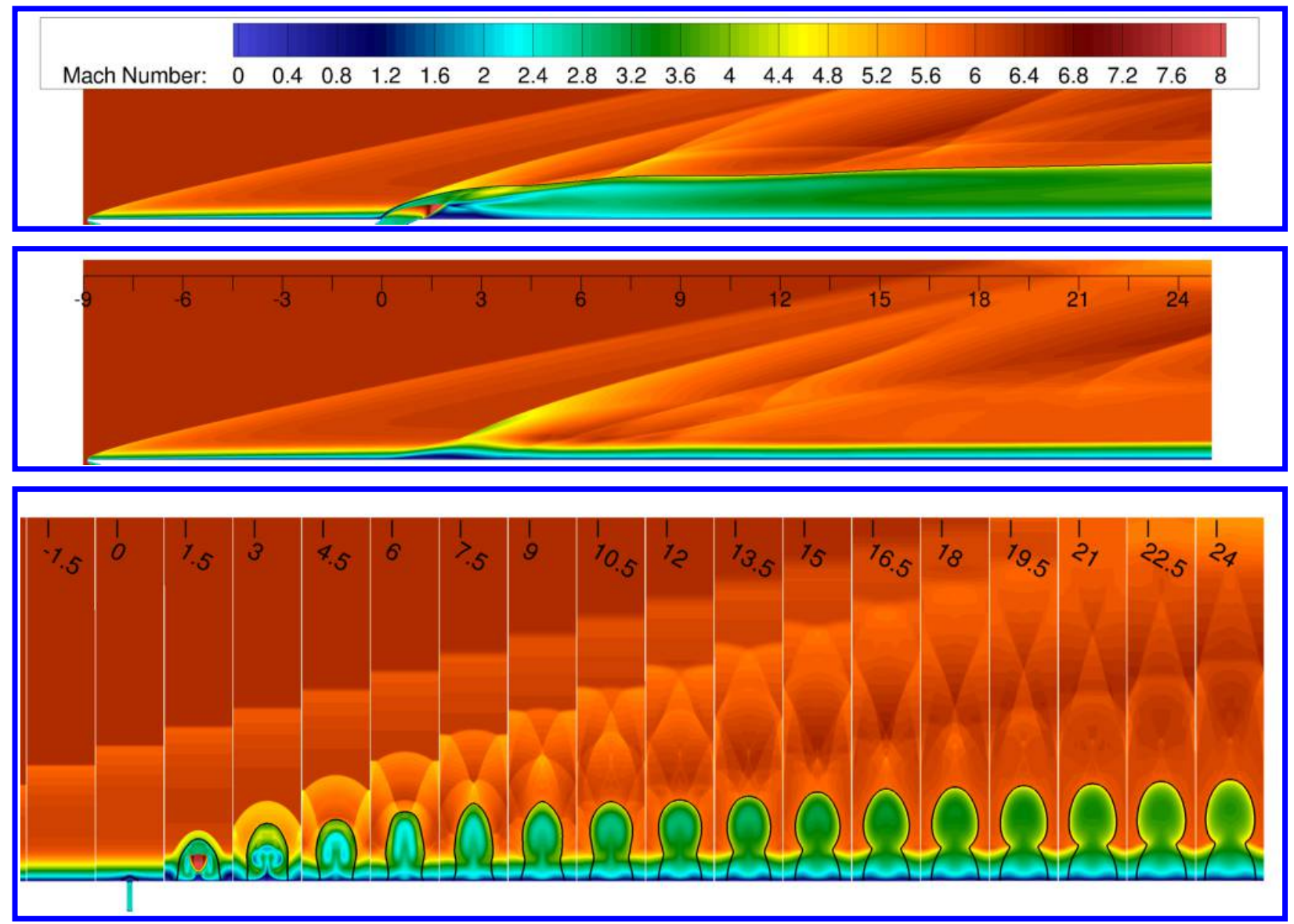

Figure 8. Contours of the Mach number on the z-planes obtained through the center of the injector port, and half-way between the injectors, and x-planes at various downstream locations for the flushwall injector simulations. Downstream distance is in inches. Black lines denote the stoichiometric value of the fuel mass fraction.

angled injection and the large-scale CVP spreads the fuel through the intended fueling area. These effects can be seen by observing the stoichiometric value of the mass fraction as it evolves in the x-planes in Fig. 7.

For the flushwall injector, shown in Fig. 8, the flow features and dynamics are somewhat similar to that of the ramp injector. An oblique bow shock wave is generated by the fuel entering into the supersonic cross-stream. The oblique shock wave angle is about 16 degrees at the injector centerline. This bow shock interacts and joins with the same bow shock wave produced by the adjacent injector fuel plumes. Similar to the shock generated by the ramp body, the flushwall injector oblique shock wave does not significantly interact with the fuel-air mixing plume but instead serves primarily to introduce a pressure difference between the top of the fuel plume and the gap between the adjacent injectors. The pressure is higher on the top of the plume, which creates a driving force to form a CVP around the fuel plume. This CVP is too weak to significantly deform the stoichiometric isosurface, nevertheless it does provide an upward lifting motion as the fuel is convected downstream. A secondary CVP also forms inside the stoichiometric isocontour line. This CVP is driven by the underexpanded fuel plume penetrating into the supersonic cross-flow and can be seen at the $\mathrm{x}=3$ station on Fig. 8. Because the flushwall injector is underexpanded (i.e., $p_{f} / p_{a}>1$, see Table 1) the fuel plume spreads laterally in addition to penetrating into the cross-flow. The underexpansion process redistributes some of the momentum (or dynamic pressure) of the fuel jet laterally, thereby reducing the amount available for penetration normal to the plate, and into the cross-flow. Nevertheless, the penetration is comparable to that of the ramp injector. 


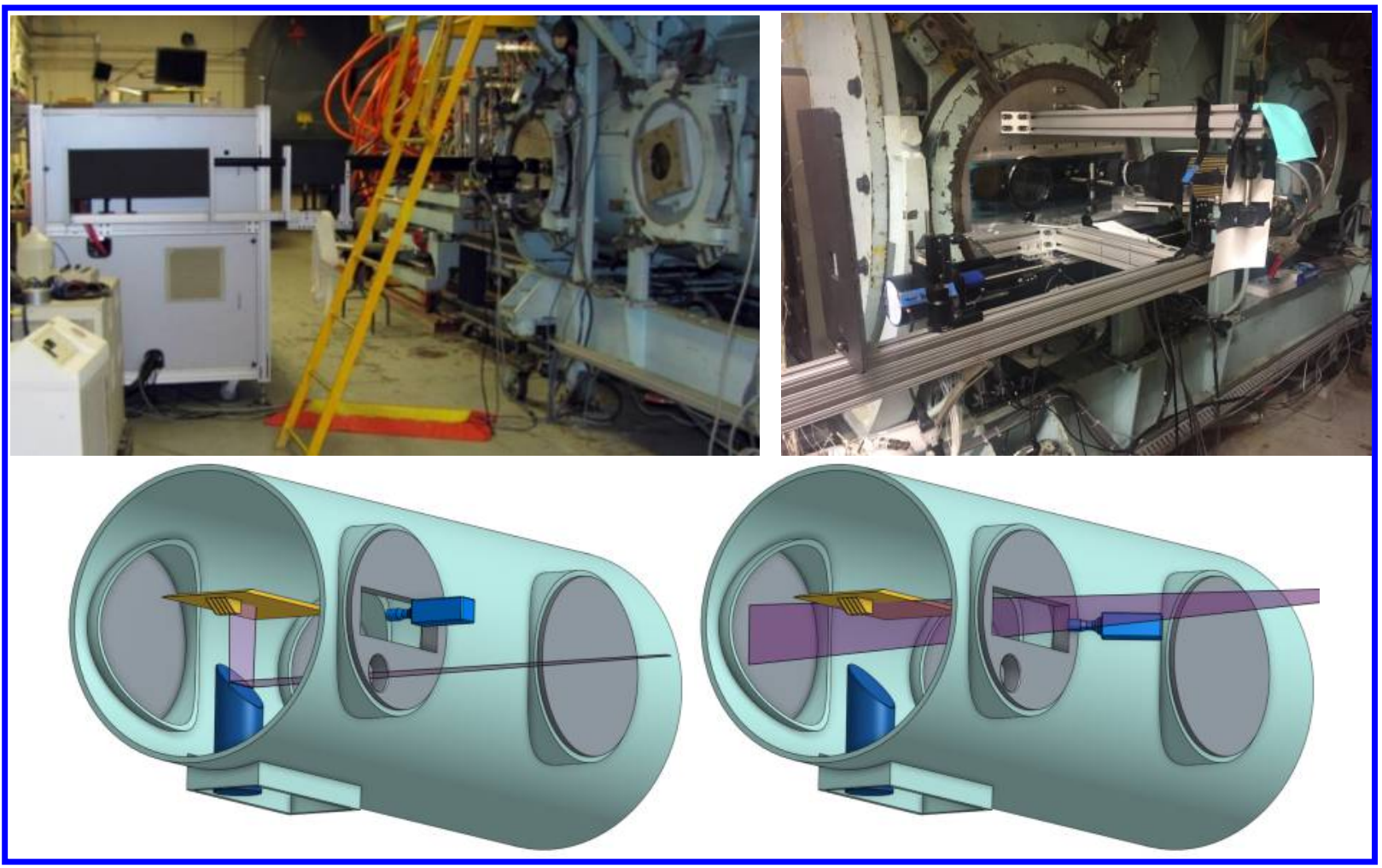

Figure 9. Photos of the Mobile PLIF cart setup (gray box on the left side of the left photo) to the left of the AHSTF (right side of the left photo) and PLIF camera and translation stage installed on the AHSTF access door (right photo). The AHSTF facility cartoons below the photos show the path of the laser sheet (violet) and the camera position (blue) during streamwise (left) and cross-stream (right) PLIF experiments.

\section{NO PLIF}

NO PLIF uses a UV laser sheet to interrogate a slice of the flow containing NO. This UV light excites fluorescence from the NO molecules, which is detected by a digital camera. One of NASA Langley's two mobile PLIF systems was installed next to the AHSTF facility as shown in Fig. 9. The two photos in Fig. 9 show the Mobile PLIF cart setup (gray box on the left side of the left photo) positioned to the left of the AHSTF facility (light blue cabin on the right side of the same photo), and the PLIF camera and the translation stage installed on the AHSTF access door (right photo). The two cartoons below the photos show the AHSTF test cabin with the facility nozzle and front bulkhead removed to reveal the experimental article, the laser path, and the camera position during the streamwise (left cartoon) and cross-stream (right cartoon) diagnostic campaigns.

The laser system uses a Spectra Physics Pro-230 Nd:YAG laser to pump a Sirah Cobra Stretch dye laser and Sirah Frequency Conversion Unit (FCU). The dye laser was operated near $624 \mathrm{~nm}$ and was sum-frequency mixed with the third harmonic of the Nd:YAG laser to produce the UV light used to excite NO. The resulting tunable laser output, near $226 \mathrm{~nm}$, was tuned to excite a variety of weak spectral lines of NO to minimize absorption. The UV laser beam passed through windows mounted into an aluminum blank manufactured to replace the facility window. The laser beam was formed into a laser sheet using a pair of lenses (usually a cylindrical lens followed by a spherical lens). High-efficiency filters (Layertec GmbH, Germany, $<1 \%$ transmission at $226 \mathrm{~nm}$ and $>80 \%$ transmission at 235-280 $\mathrm{nm}$ ) were used to transmit the LIF signal while rejecting the laser scatter. The same cameras, excitation schemes, etc., were used in other past experiments. ${ }^{18,19}$ The fluorescence was imaged onto a gated, intensified charge-coupled device (CCD) (Princeton Instruments PIMAX-II ${ }^{\circledR}$ ), and represented using 16-bits of resolution. A single Nikon ${ }^{\circledR}$ UV lens with a focal length of $105 \mathrm{~mm}$ and $\mathrm{f} / 4.5$ aperture was used. The camera magnifications were measured by imaging a regular pattern of dots of known spacing (known as a dotcard), with the dotcard placed in the image plane. The images were obtained at a rate of $10 \mathrm{~Hz}$ with flow-stopping time resolution ( $<1$ microsecond). The final images 
were also postprocessed as discussed in the next section. The spatial distribution of intensity in the laser sheet was not monitored, but could be inferred from uniform flow regions obtained in the images.

Three different sets of LIF transition lines are used in the current work. In terms of Hund's Case A notation ${ }^{38}$ these are: ${ }^{\mathrm{S}} \mathrm{R}_{21}(9.5)+\mathrm{Q}_{1}(20.5)+{ }^{\mathrm{Q}} \mathrm{P}_{21}(20.5)+\mathrm{R}_{2}(18.5),{ }^{\mathrm{O}} \mathrm{P}_{12}(6.5)+{ }^{\mathrm{O}} \mathrm{P}_{12}(14.5)$, and ${ }^{\mathrm{R}} \mathrm{Q}_{21}(12.5)+\mathrm{R}_{1}(12.5)$, where the number in the parenthesis denotes the rotational quantum number. The laser linewidth is about $2 \mathrm{~cm}^{-1}$ for the first set of lines, and $0.1 \mathrm{~cm}^{-1}$ for the latter two. Each set of lines exhibits different sensitivity to pressure, temperature, and mole fraction of $\mathrm{NO}$, and has different absorption characteristics. The latter two transition lines have been selected for their linear correlation with either mass fraction or mole fraction of helium, respectively. That is, the LIF signal corresponding to these two sets of lines correlated close to linearly with mass or mole fraction of helium in a priori tests using LIF modeling software LINUS ${ }^{38}$ under the nominal pressure, temperature, and NO concentration conditions representative of those found in the current experiments. In addition, because, in the current experiments, the NO is present in the facility air and the fuel simulant is "unseeded" (contains no NO), the dark, no-signal regions, are representative of the fuel stream, and conversely, bright regions contain air. This approach, although convenient from an experimentation point of view because NO is naturally produced in the facility arc-heater, makes the LIF more susceptible to the attenuation of signal due to absorption because the laser light has to pass through about 2 feet of the test cabin filled with NO-containing air en-route to the PLIF imaging region. Apriori estimates of absorption through the test cabin using LINUS ${ }^{38}$ under expected tunnel operating conditions suggest that up to $75 \%$ laser energy could be absorbed before reaching the field of view of the CCD camera.

\section{PLIF Image Postprocessing}

The instantaneous, raw PLIF images typically require some amount of postprocessing. The corrections can include manipulations that do not alter the raw value of the PLIF signal, such as image magnification adjustments, and/or perspective control (unwarping), and those that alter the value of the PLIF signal, such as adjustments to the signal "zero" reference, corrections for laser sheet and shot-to-shot intensity variations, and image-intensity-based corrections for laser sheet absorption. The final postprocessing step may also include time averaging to allow for comparisons with time-averaged simulation data. In the current work, Image ${ }^{39}$ software is used for all image postprocessing and analysis. An example of a few of the steps are shown in Figs. 10-13. Figure 10 shows the raw and unwarped cross-stream PLIF images along with the raw and target dotcards used in the correction process of the mixing flowfield 0.5 inches downstream of a strut injector. The unwarping effectively involves computing a transformation function that maps the pixels from the dotcard imaged at the laser sheet location to the target dotcard viewed straight-on. This transformation function is then applied to all of the raw PLIF images to obtain the corresponding unwarped images. Figure 11 shows the unwarped but otherwise yet uncorrected PLIF image of the same strut injector, and the corresponding image corrected for left-to-right laser sheet absorption. Plots of the PLIF intensities for the raw and corrected images versus distance in pixels measured in the direction of laser sheet absorption along the bottom few pixels of each image (i.e., away from the mixing regions) are shown to the right of the images. The raw image exhibits about a factor of two to four attenuation of the PLIF signal as the laser light is absorbed by the NO molecules. The image intensity-based correction procedure can only partially correct for this effect because the nominal absorption intensity is based on localized left-to-right intensity variation, although in the current image the correction is quite effective. However, it should also be noted that the signal-to-noise ratio decreases from left-to-right as a result. The hills and valleys visible in the line plots are a result of the expected sensitivity of the PLIF signal to both pressure and temperature, and no attempt is made to correct for these variations. Figure 12 shows a single streamwise PLIF image before and after full postprocessing, and the mean value of the PLIF signal computed from the central lower half portion of every image versus the image number. These plots indicate that before postprocessing the shot-to-shot PLIF signal intensity can vary by as much as a factor of two. These variations are due to both, the laser sheet intensity variations as well as the time-varying amount of NO produced by the facility. The applied corrections significantly reduce these variations allowing for construction of more equally weighted averages for comparisons with time-average simulation data. The spike in the mean image intensity values occurring for corrected images ranging from about 420 to 460 is due to the loss of signal to a portion of the image due to the motion of the laser sheet that gets amplified in a correction process. These images were not used in averaging of the streamwise PLIF data. Finally, Fig. 13 shows the time-average of 30 frames $(3.0 \mathrm{sec}$.) of the postprocessed cross-stream PLIF images. All corrections discussed above have been applied to the individual image frames before averaging. Notable in the image is the increasing level of blurriness when moving away from the central strut injector. This loss of focus is a result of imaging at a relatively high angle of incidence to the laser sheet, and can also be observed in the dotcard image of Fig. 10(b). Although the perspective can be corrected, 


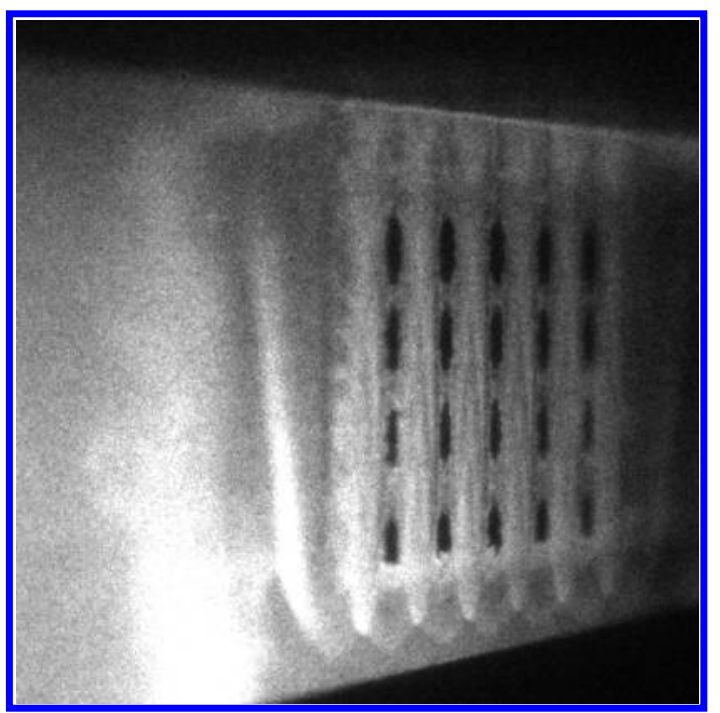

(a) Unaltered, raw, cross-stream PLIF image of the mixing flowfield downstream of a strut injector.

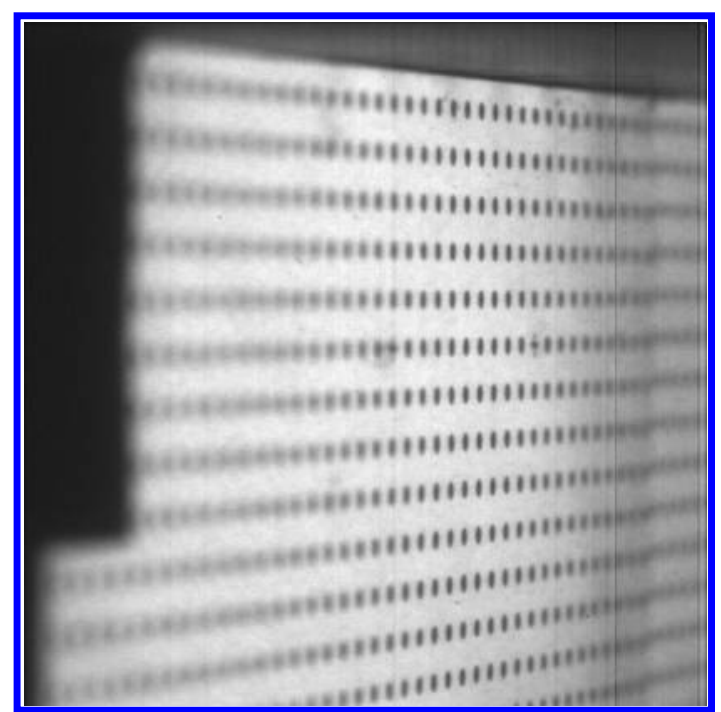

(b) Dotcard image taken at the location of the laser sheet.

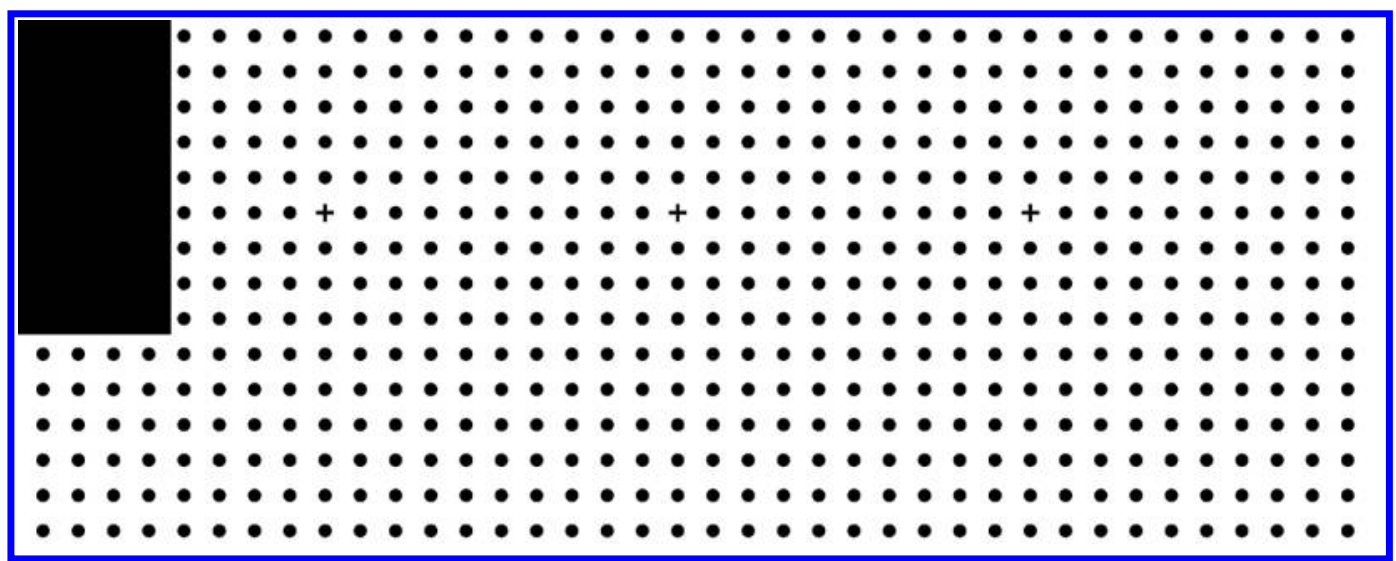

(c) Undistorted target dotcard used for the image in (b).

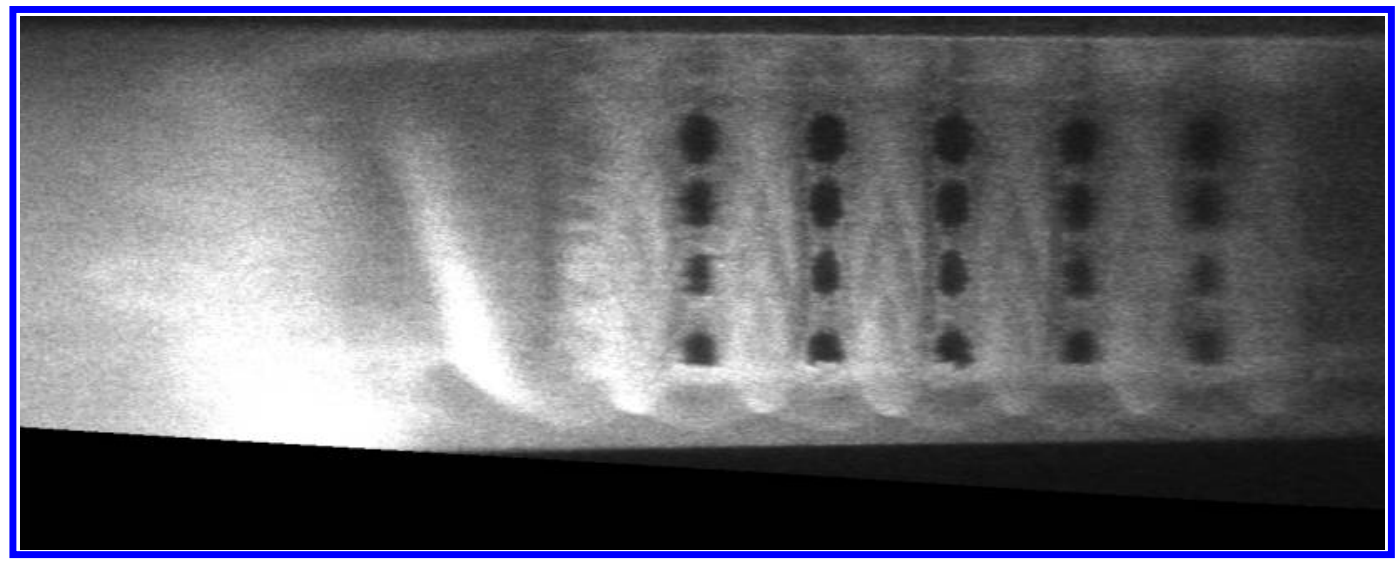

(d) Corrected (or unwarped) image corresponding to (a).

Figure 10. Raw PLIF image, distorted and undistorted dotcards, and the corresponding corrected (unwarped) PLIF image at 0.5 inches downstream of a strut injector. 


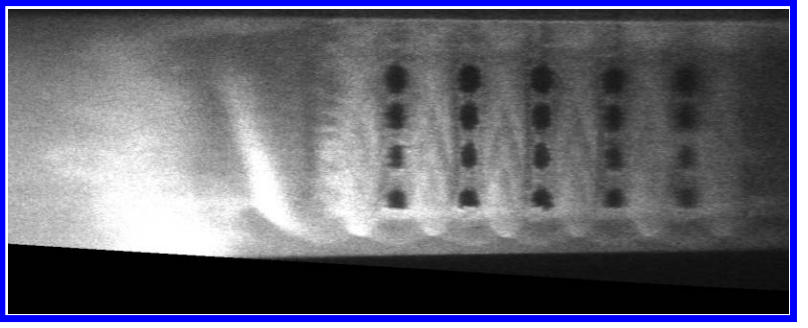

(a) Unwarped, raw PLIF image

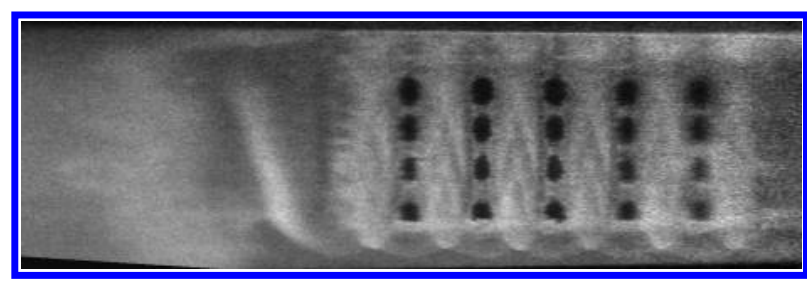

(c) Unwarped, postprocessed PLIF image

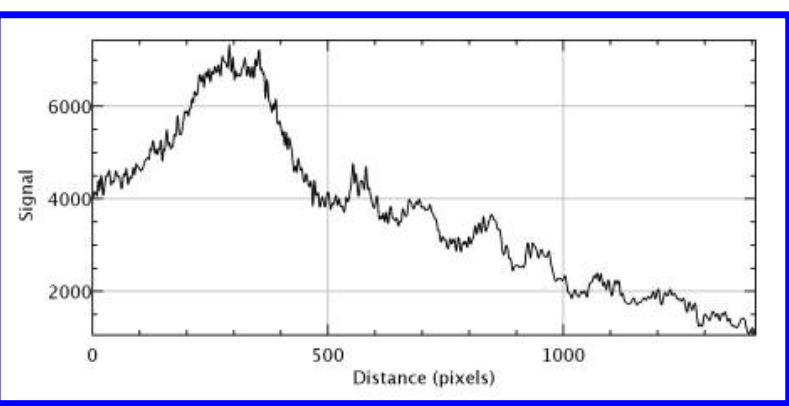

(b) Raw PLIF signal

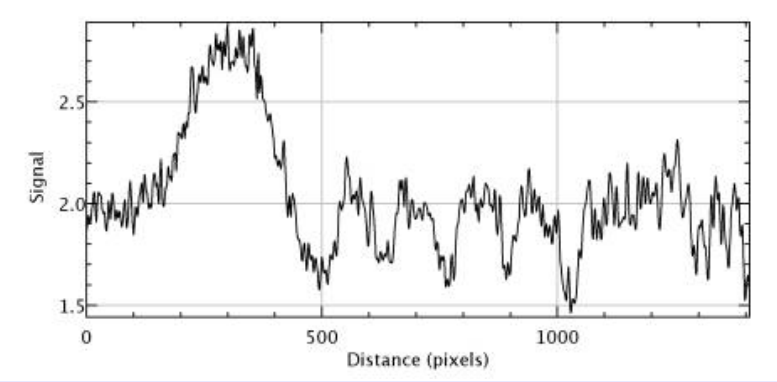

(d) Postprocessed PLIF signal

Figure 11. Unwarped raw and postprocessed cross-stream images of the mixing flowfield downstream of the strut injector and corresponding PLIF signal vs. left-to-right image pixel count.

the loss of focus cannot. However, in the future experiments, a Scheimpflug principle could be adopted to improve the focusing when performing cross-stream PLIF imaging at high angle of incidence.

The run-to-run variability in the PLIF imaging can be visually examined in Fig. 14. The rows (from top to bottom) show time-average cross-stream images obtained at several downstream location from the ramp injector face, while the left and right columns show the data obtained from two successive runs. The data was obtained by tuning the laser to mass fraction sensitive LIF lines. The first two rows show the fuel-off and -on condition, respectively, at 0.5 inches downstream of the injector face, followed by fuel-on imaging at 1.0, 2.0, and 4.0 inches downstream. Although the images from different runs are nearly indistinguishable, notable in the imaging is a band of strong absorption that could not have been corrected by the current image postprocessing approach. Figure 15 also shows run-to-run variability in the PLIF imaging, however, this time the data was obtained about eight months apart. The images on the left were obtained eight months prior to those on the right. The locations are the same as those above, however, this data was obtained using the mole fraction, instead of mass fraction, sensitive LIF lines. As those in Fig. 14, these images are qualitatively similar indicating robustness and good repeatability characteristics of both the facility and the current PLIF approach.

\section{Fluorescence Modeling}

The PLIF signal level $S$ (number of counts recorded on a pixel of a detector) is a function of temperature, pressure, mole-fraction and a number of known experimental parameters. A model for the fluorescence signal level in the weak fluorescence regime is given by Paul et al. ${ }^{40}$ :

$$
S \propto \frac{\chi_{N O} P}{k_{B} T} \phi \sum_{i}\left(f_{B} B_{12} g\right)_{i}
$$

where $\chi_{N O}, P, T, k_{B}, f_{B}, B_{12}$, and $g$ are the mole fraction of NO, mixture pressure and temperature, Boltzmann constant, Boltzmann fraction, Einstein absorption coefficient, and spectral overlap integral, respectively. The ratio $\phi$, 


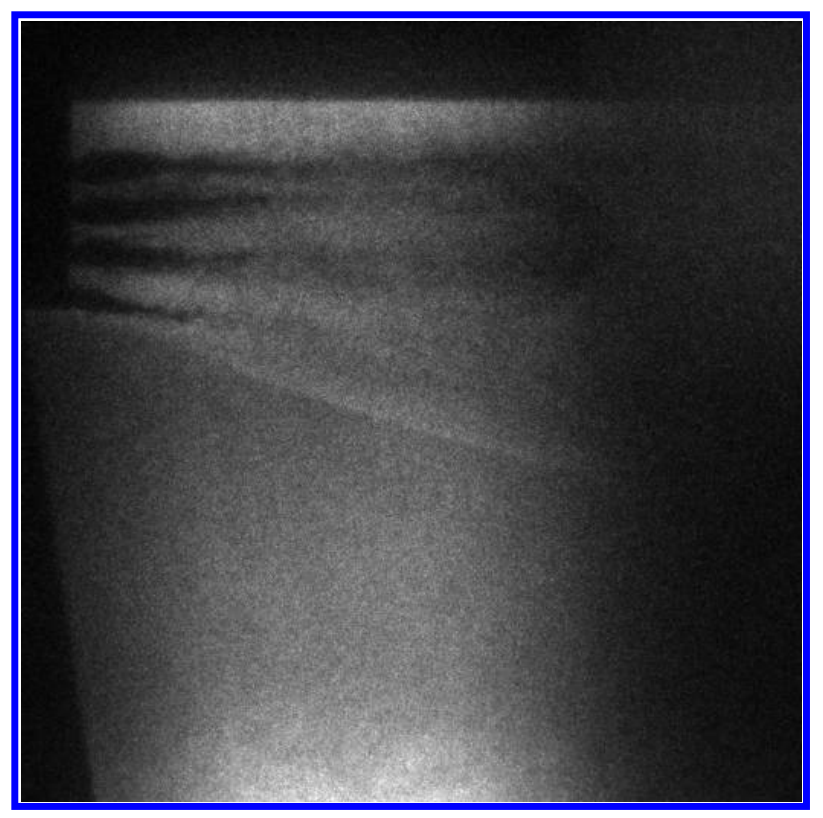

(a) Raw PLIF image

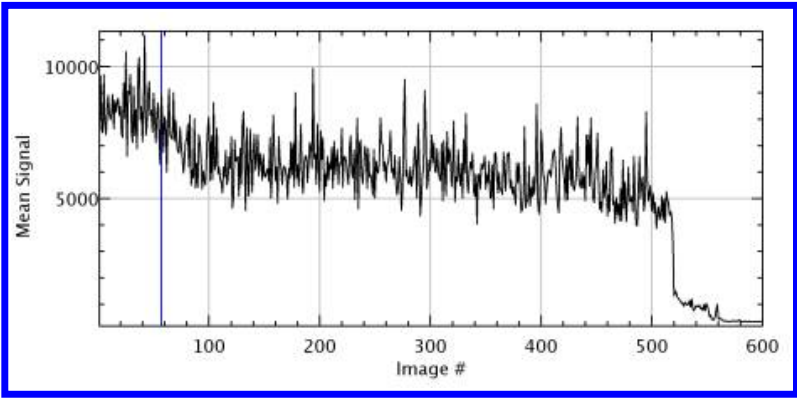

(c) Raw PLIF signal

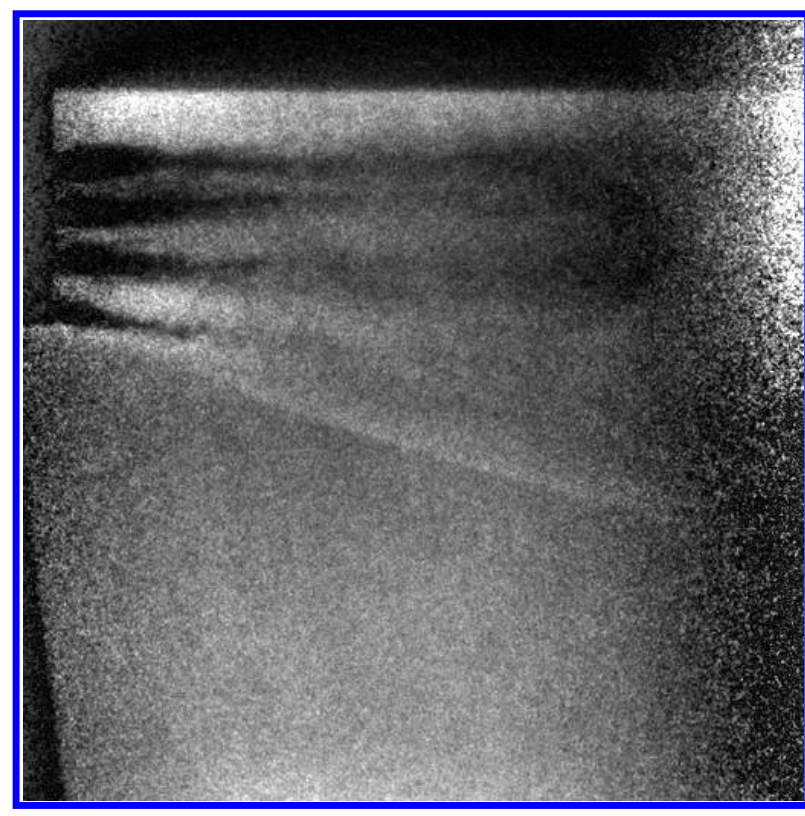

(b) Postprocessed PLIF image

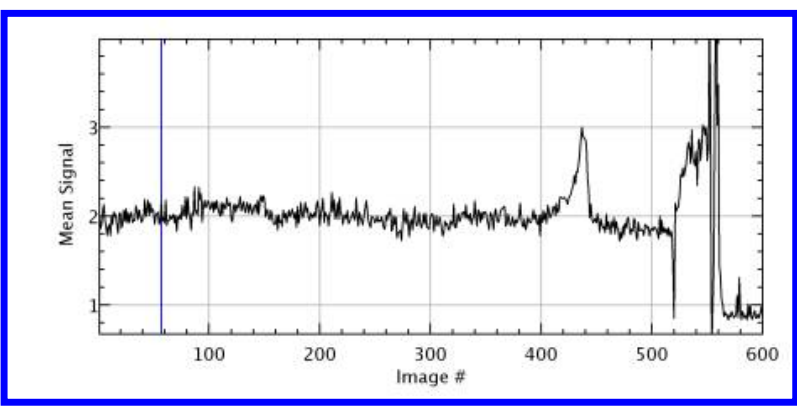

(d) Postprocessed PLIF signal

Figure 12. Raw and postprocessed PLIF images and corresponding PLIF signal vs. image number.

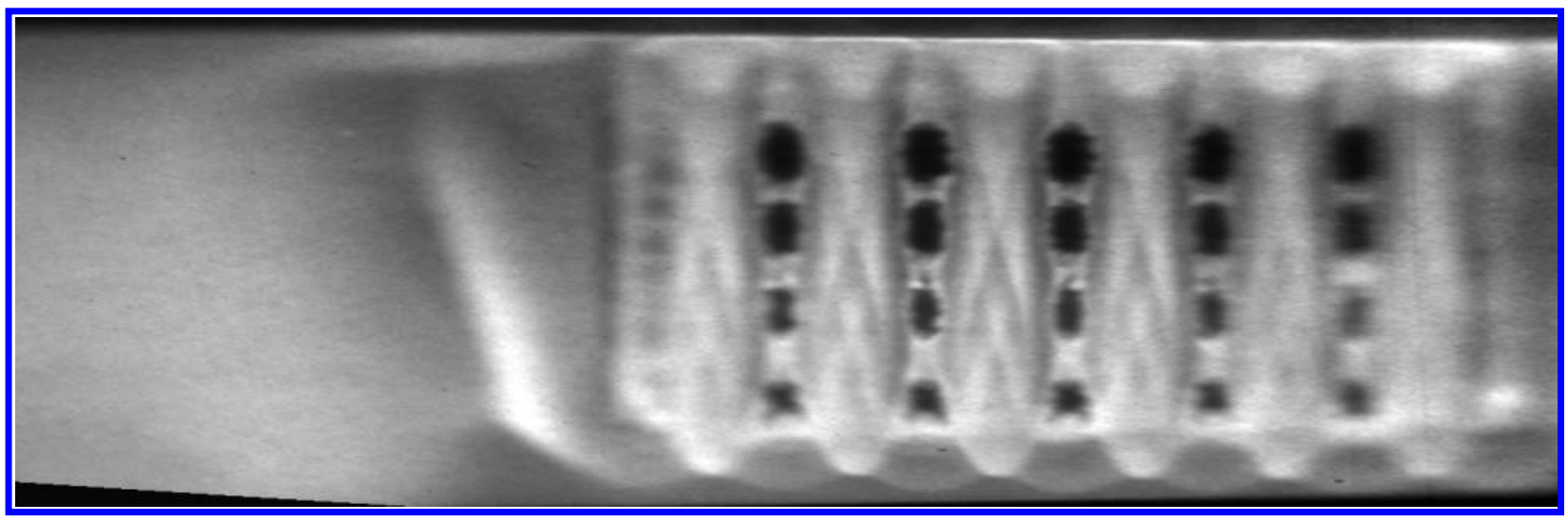

Figure 13. Time average over about 30 frames ( 3.0 seconds) of the postprocessed cross-stream images for the strut injector at $\mathrm{x}=0.5$ inches. 


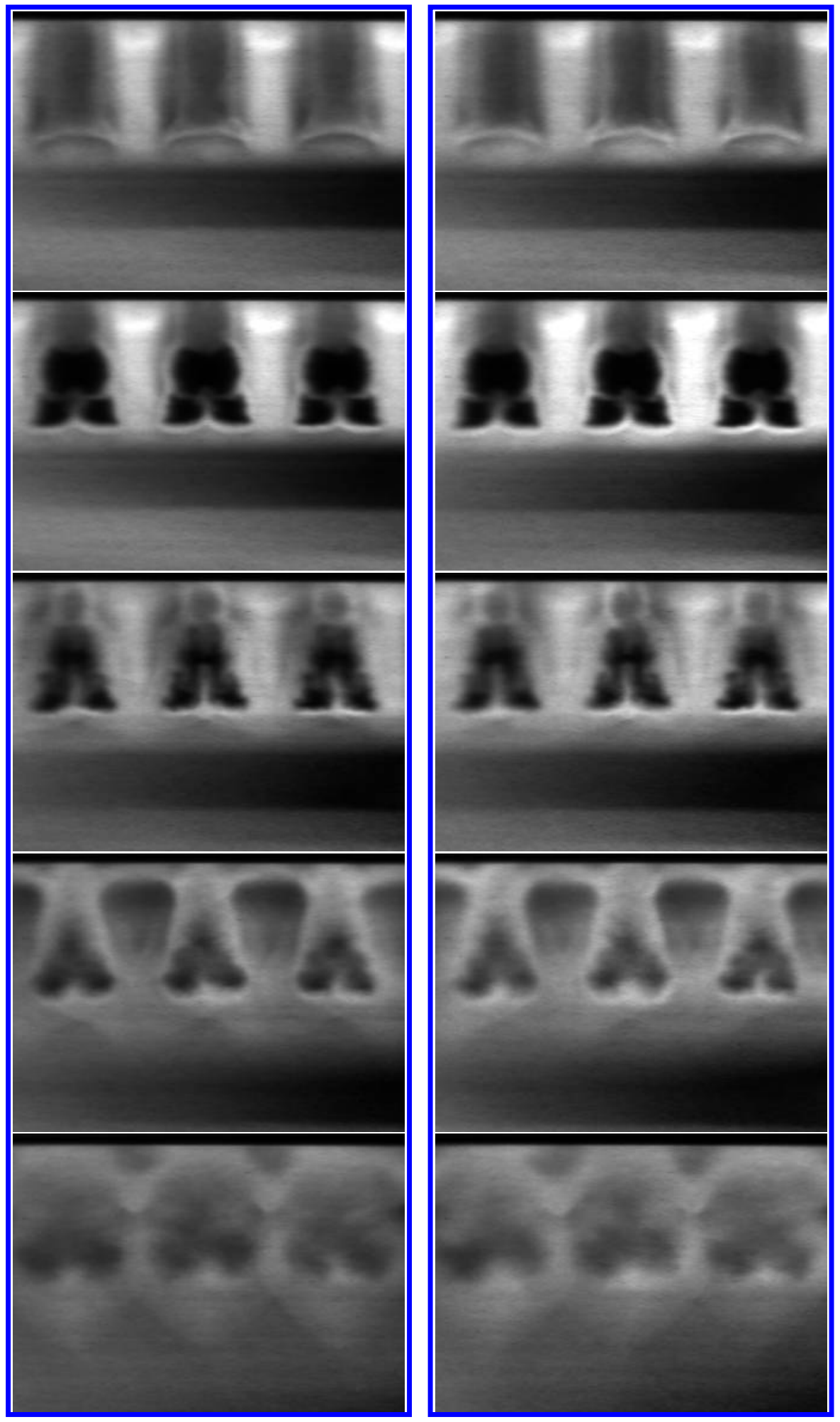

Figure 14. Time-averaged cross-stream PLIF images for the ramp injector for two different sequential experimental runs at the same nominal conditions and obtained using mass fraction sensitive LIF lines. The images are located at (from top to bottom): $0.5,1.0,2.0,4.0$ inches downstream of the injector face, with the first two rows showing fuel off and on condition, respectively, at 0.5 inches. 


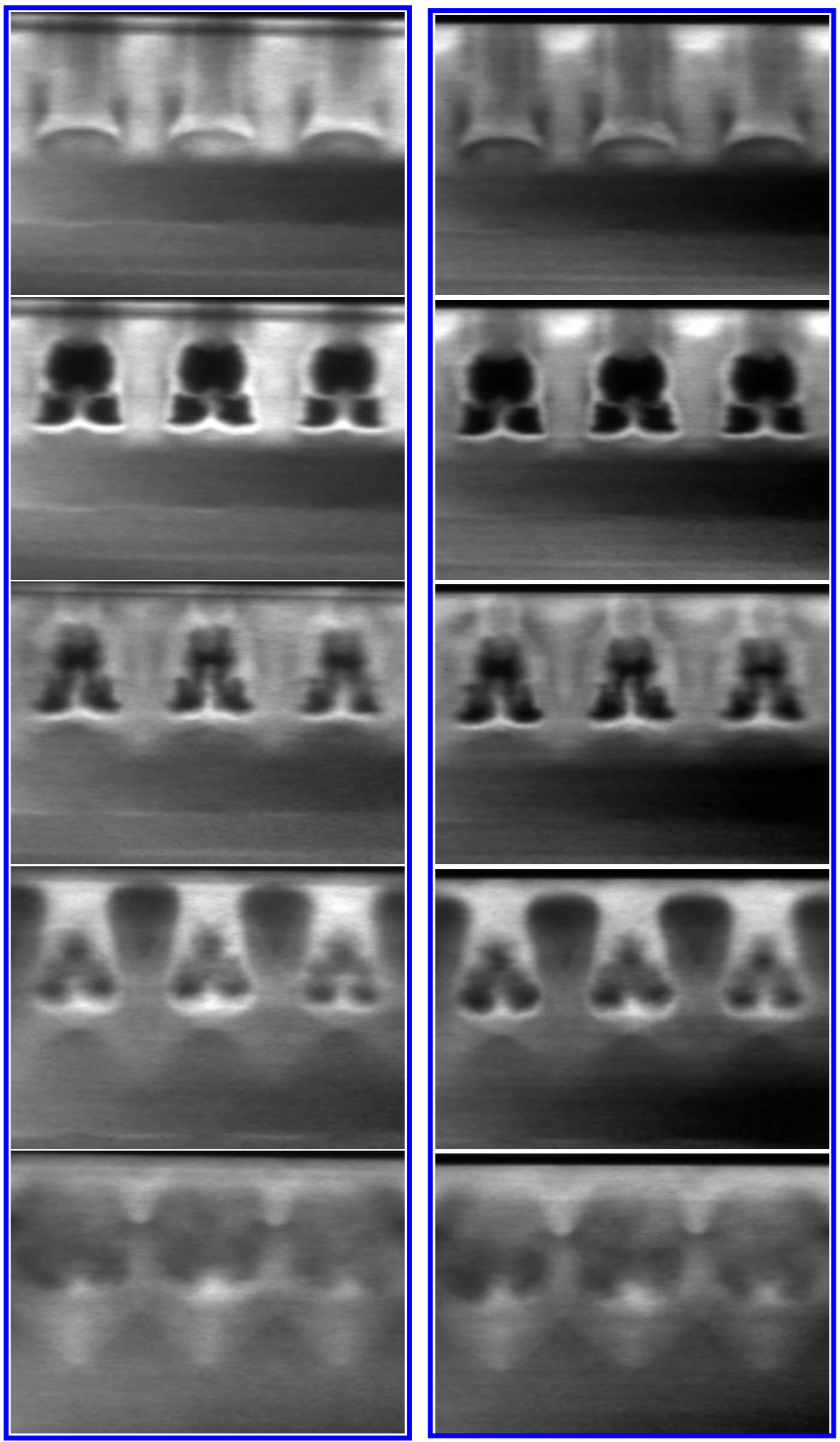

Figure 15. Time-averaged cross-stream PLIF images for the ramp injector for two different experimental runs performed about eight months apart at the same nominal conditions and obtained using mole fraction sensitive LIF lines. The images are located at (from top to bottom): $0.5,1.0,2.0,4.0$ inches downstream of the injector face, with the first two rows showing fuel off and on condition, respectively, at 0.5 inches. 
defined as

$$
\phi=\frac{A_{21}}{A_{21}+Q},
$$

is the fluorescence yield, where $A_{21}$ and $Q$ are the Einstein spontaneous emission coefficient and quenching rate, respectively. Equation 1 contains nonlinear dependencies on pressure, temperature, and mole fraction of NO through the Boltzmann fraction, the spectral overlap integral, and the quenching rate. In addition, the spectral overlap integral also accounts for signal attenuation due to any laser "detuning" from the intended transition line, and the Doppler effect. All quantities are modeled following the approach of Ivey et al. ${ }^{19}$ with the model constants and coefficients obtained from LINUS. ${ }^{38}$ The above model was applied to the CFD data to obtain CFIs equivalent to those obtained experimentally with PLIF. However, it should be noted that the LIF model used herein has not been formally validated, makes Gaussian assumptions on the shapes of the spectral laser and absorption profiles (i.e., triple-Gaussian assumption ${ }^{19}$ ), and neglects the attenuation of the laser light intensity caused by absorption of NO. Under some circumstances, the latter effect can be partially corrected by the image post-processing, as noted during the PLIF image postprocessing discussion. However, for the current experiments, any off-image absorption due to the laser light passing through the NO-filled test cabin can not be corrected and is difficult to quantify. Furthermore, for those images where absorption could not be effectively corrected in post-processing (e.g., Fig. 14) because various rates of absorption are observed in the image, the Beer-Lambert law ${ }^{41,42}$ could be used to model some of the effect. This work defines the signal attenuation parameter, $D$, which is the ratio of transmitted to incoming LIF signal, as:

$$
D=\frac{S_{t}}{S_{i}}=C_{1} \operatorname{Exp}(-\tau)
$$

where $S_{i}, S_{t}$, and $\tau$ are the incident signal, transmitted signal, and the optical depth, respectively. The optical depth is further defined as:

$$
\tau=C_{2} \int_{0}^{l} S(z) d z
$$

where the integral is evaluated over the optical path. The above equations contain two constants $C_{1}$, and $C_{2}$, which can be adjusted to tune the level of absorption in CFIs to that visually observed in the experiment. In the current work, the CFI image intensity (i.e. black-to-white is zero-to-one) was used as a surrogate for the PLIF signal, $S$, with constants $C_{1}$, and $C_{2}$, set to one, and varied between 1 to 3, respectively. Such adjustments were needed because the current CFD assumes that an infinitely wide array of injectors is a good representation of the finite-width experimental article. Therefore, even a more complex and quantitative absorption model, if applied here to obtain the CFI, would still require some tuning to account for absorption in the unmodeled cabin portion of the flowfield.

\section{Results and Discussion}

Figures 16-20 show the comparisons between the time-averaged PLIF images and the corresponding computationally derived PLIF, termed CFI, obtained for different fuel injectors and using different sets of LIF transition lines. Figure 16 shows the time-averaged streamwise images obtained along the centerline of the strut injector block and cutting through the middle injector. The injector body is partially visible in the top-left section of the images with the dark regions representing fuel plumes emanating from the four injector body fuel ports. The laser sheet travels from bottom to top of the image. As expected, both the experiment and the CFI are sensitive to not only the NO concentration (recall that there is no NO in the fuel simulant stream) but also to temperature and pressure. Both images are obtained by probing, and modeling the LIF from, the ${ }^{\mathrm{S}} \mathrm{R}_{21}(9.5)+\mathrm{Q}_{1}(20.5)+{ }^{\mathrm{Q}} \mathrm{P}_{21}(20.5)+\mathrm{R}_{2}(18.5)$ transition lines. The qualitative comparison appears reasonable with many of the flow features visible in both the PLIF images and the CFI. No absorption modeling is applied to the CFI here because little is observed in the post-processed PLIF. The CFI generally appear in greater contrast as compared to the PLIF. One reason for this may be that steady RAS assumes that all fluctuations are turbulent in nature and lacks the "smoothing" effects associated with time-averaging of intrinsically unsteady large or discreet flow features. This effect is, in general, most pronounced for the discrete flow features like shocks. In addition, although the pixel-by-pixel image resolution is the same between the PLIF and CFI, the resolution of the streamwise plane in the CFD, on which the CFI is computed, is significantly greater than that of the PLIF CCD camera. The darker image regions that are the fuel plumes also appear to persist further downstream in the CFI than PLIF image. This would seem to indicated a greater degree of mixing in the experiments than CFD. However, this slight discrepancy could also be the result of modeling errors in the CFI. 

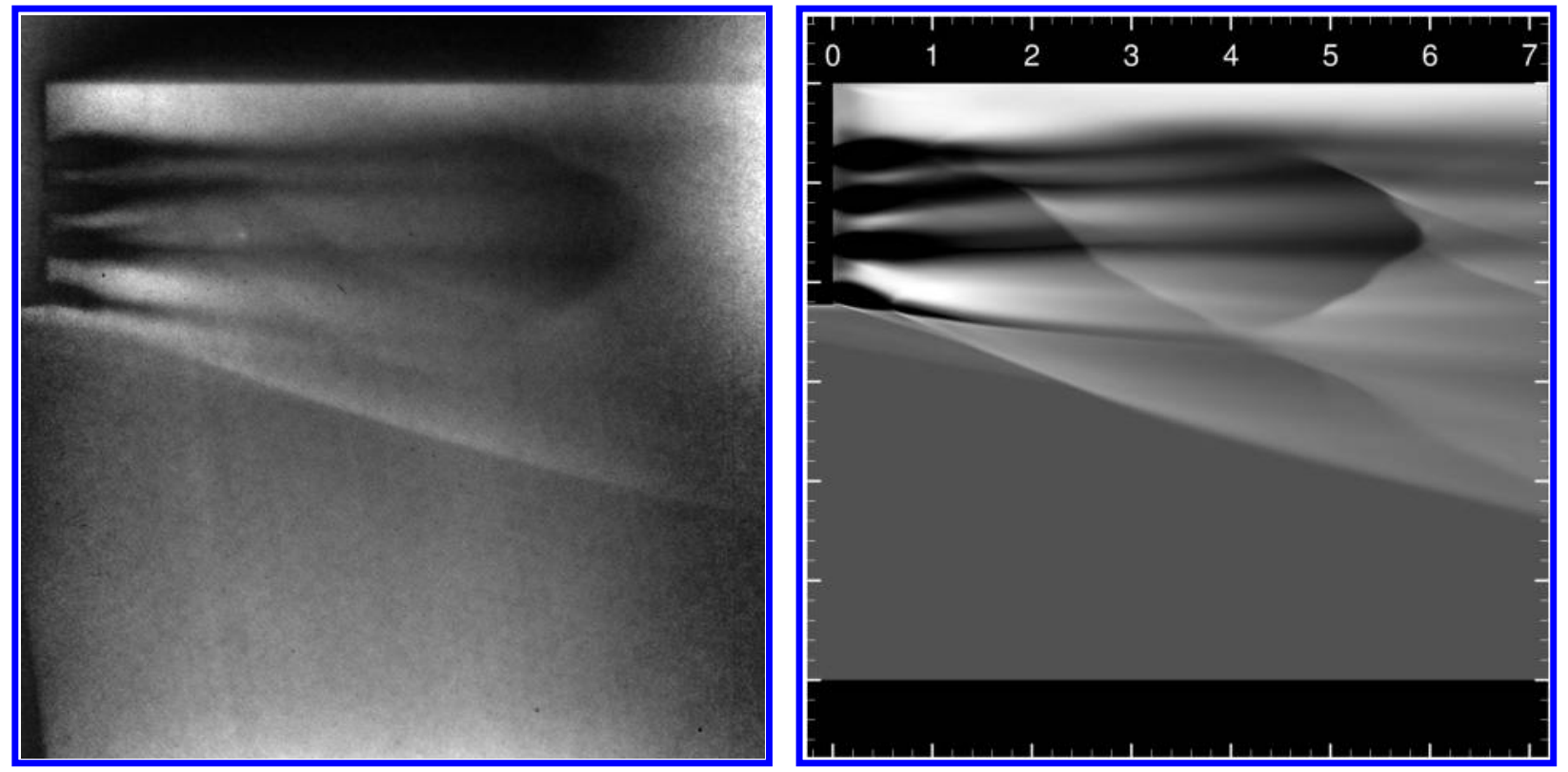

Figure 16. Time-averaged streamwise PLIF image (left) through the center of the strut injector and the corresponding CFI (right). The dimensions are in inches.

Figure 17 shows several time-averaged cross-stream PLIF images (left-most column), CFI (two middle columns), and the mass fraction of air (right-most column) obtained for the strut injector. The image planes are located at 0.5, 1.0, 2.0, 4.0, and 6.0 inches downstream from the injector face, respectively. The laser sheet travels from left-toright in these images. It should also be noted that the PLIF images have been cropped here to focus on the injector flowfield and match the visual extent of the CFI. The PLIF and the CFI are obtained using the mass fraction sensitive, ${ }^{\mathrm{O}} \mathrm{P}_{12}(6.5)+{ }^{\mathrm{O}} \mathrm{P}_{12}$ (14.5), transition lines. The left and right central columns of the CFI show images with the laser tuned to the 14.5 and 6.5 lines, respectively. The intent of the diagnostic approach was to tune the laser to the 6.5 line to improve the correlation of the LIF with the mass fraction of helium (shown on the right-most column). However, because these lines are positioned within one full width of max height (FWMH) of the laser linewidth from one another, and have grossly different rotational quantum numbers and absorption characteristics, tuning of the laser to a specific line proved challenging during the experiments. Since there is no (or little) Doppler-shift effect present in the PLIF images, it can be safely assumed that the laser is indeed well-tuned to one of these lines, and so it is possible that the laser was actually tuned to the 14.5 line in the experiments. The CFI for this latter line produces a much closer visual match to the PLIF and a reasonable overall qualitative agreement. It should be noted that it is also possible that the CFI modeling at the lower values of the rotational quantum numbers introduces significant errors into the CFI.

Some shockwave patterns visible in the gaps between the injectors at 0.5 and 1.0 inch locations appear different between the PLIF images and the CFI. This could be the result of slight differences in the facility air Mach number and the ratio of specific heats between the experiment and the CFD (recall that CFD was performed pretest). Some "edge" effects are also visible in PLIF images at the farthest downstream location. These manifest themselves through the distortions to the shock patterns near the left and right edges of the images. These edge effects are absent from the CFI, which utilize symmetry boundary conditions and effectively simulate an infinite row of injectors.

Figure 18 shows several time-averaged cross-stream PLIF images (left-most column), CFI (two middle columns), and the mass fraction of air (right-most column) obtained for the ramp injector. The downstream locations are the same as before. The PLIF and the CFI are also obtained using the mass fraction sensitive transition lines, and the left and right of the two central columns of the CFI show the effect of tuning the laser to either the 14.5 and 6.5 lines, respectively. Similar to above, the CFI of the LIF signal corresponding to the 6.5 line dominates exhibits significantly less sensitivity to pressure and temperature, and makes the LIF signal compare well visually with the mass fraction of air shown in the right-most column. However, once again it is the CFI corresponding to the 14.5 line, that produces closer visual match to the PLIF. The qualitative agreement between the PLIF and latter CFI is similar to that observed for the strut injector. However, notable in the PLIF images is the absorption of signal in the shock layer just below 


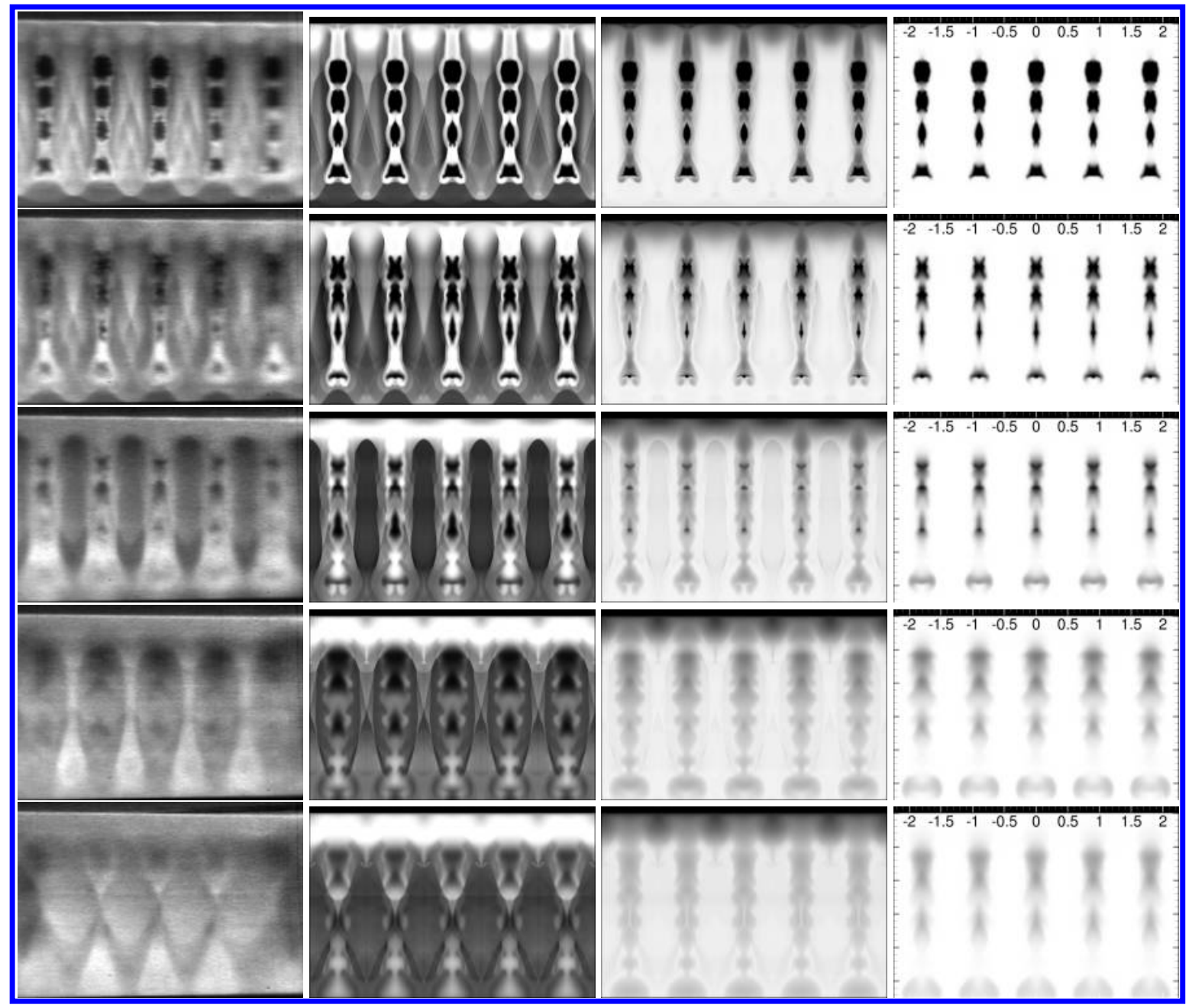

Figure 17. Time-averaged cross-stream PLIF images (left-most column) for the strut injector, the corresponding CFI (two middle columns), and mass fraction of air (right-most column) at $0.5,1.0,2.0,4.0$, and 6.0 inches (top-to-bottom) downstream from the injector face. The two middle CFI are obtained using the mass fraction sensitive LIF transition lines, with the left set of images corresponding to LIF where some laser detuning was allowed. The dimensions are in inches.

the ramp injector bodies. This absorption is a result of rapid depletion of the light energy due to the increase in both temperature and pressure inside the shock layer. The absorption observed in this shock layer was not corrected (or only partially corrected) by the PLIF image postprocessing because its rate is greater than that for which the image postprocessing was adapted to. Although, the absorption effect is unmodeled in the CFI, it could be approximated using the Beer-Lambert law as shown next.

Figure 19 also shows several time-averaged cross-stream PLIF images (left-most column), CFI (two middle columns), and the mole fraction of air (right-most column) obtained for the ramp injector. The downstream locations are the same as before. However, the PLIF and the CFI are obtained using the mole fraction sensitive transition lines: ${ }^{\mathrm{R}} \mathrm{Q}_{21}(12.5)+\mathrm{R}_{1}(12.5)$. These "double lines" are easier to tune to, and are not sensitive to small laser detuning because they are much closer to each other in the spectral space (about $0.04 \mathrm{~cm}^{-1}$ ) and have the same rotational quantum number. The left of the two central columns of the CFI includes a simple absorption model as described in the fluorescence modeling section above. This model does qualitatively reproduce the left-to-right laser light absorption pattern. However, although the level of attenuation is proportional to signal strength, the entire CFI is clearly darker left-to-right, whereas the PLIF images seem only to contain absorption in the shock layer below the ramp injector body. This is somewhat expected because the absorption in the PLIF images have been partially corrected by the image postprocessing approach. Furthermore, the left-to-right absorption pattern in the CFI is also similar to the ab- 


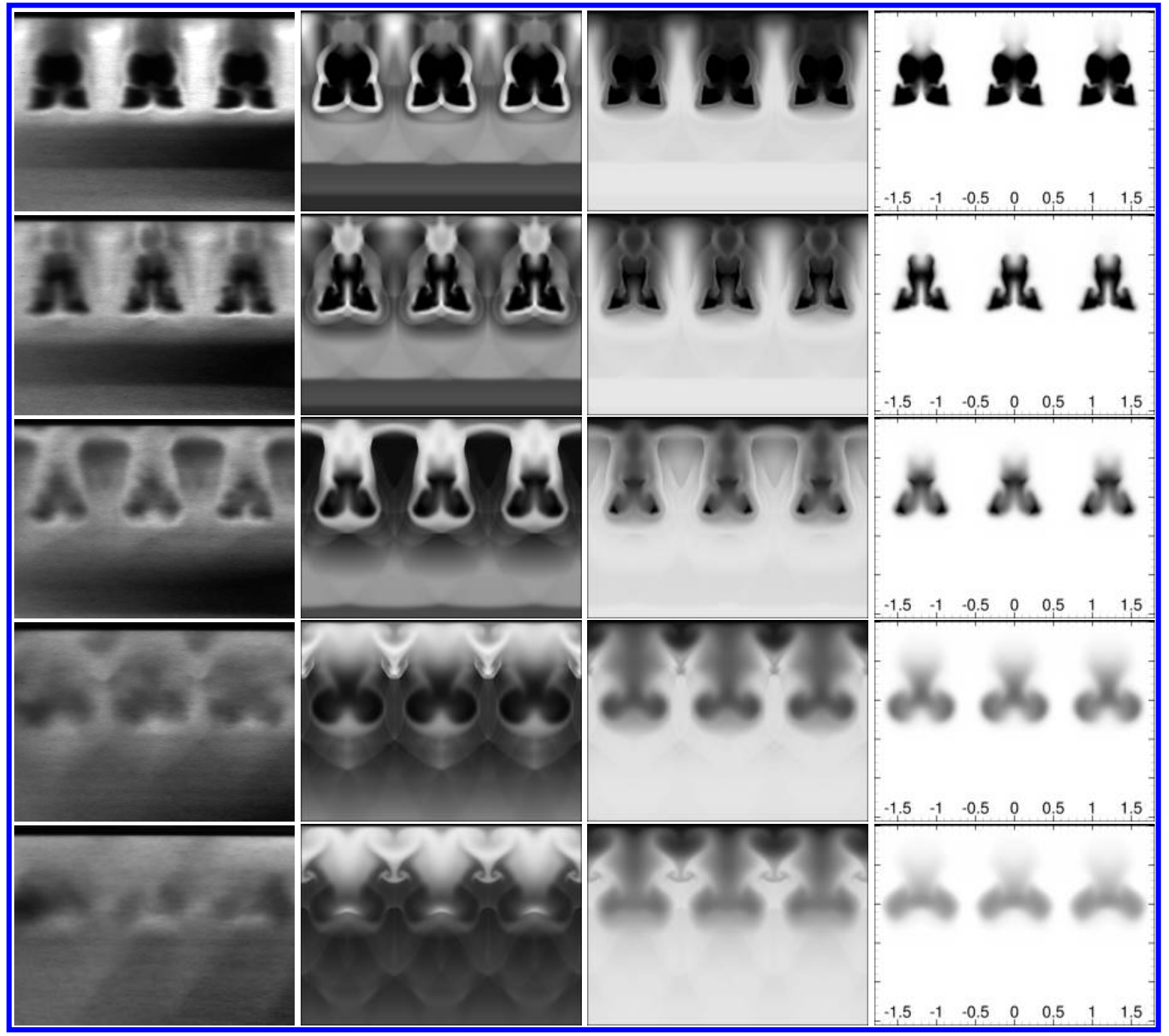

Figure 18. Time-averaged cross-stream PLIF images (left-most column) for the ramp injector, the corresponding CFI (two middle columns), and mass fraction of air (right-most column) at $0.5,1.0,2.0,4.0$, and 6.0 inches (top-to-bottom) downstream from the injector face. The two middle CFI are obtained using the mass fraction sensitive LIF transition lines, with the left set of images corresponding to LIF where some laser detuning was allowed. The dimensions are in inches.

sorption patterns observed in the raw PLIF images (e.g., Fig. 11). The CFI without the absorption model is also shown in the right of the two central columns. Because the fuel simulant (helium) molecules are lighter than air, the mole fraction of helium in the air mixture persists at high values downstream as compared to the mass fraction. This can be directly noted in the contours of the mole fraction of air, where the dark regions representing helium persist further downstream. Although the mole fraction sensitive lines also exhibit significant pressure and temperature sensitivity, it is still observed that the fuel plume shape and size are well correlated with the mole fraction of air. The qualitative agreement between the PLIF and CFI is similar to that observed above.

Figure 20 shows several time-averaged cross-stream PLIF images (left-most column), CFI (two middle columns), and the mole fraction of air (right-most column) obtained for the flushwall injector. The image planes are located at $-1.0,-0.62,-0.12,0.38,1.38,2.38,3.38$, and 4.38 inches (top-to-bottom) from the trailing edge of the injector, respectively. Because the injector is 1.113 inches long, all the negative values represent planes that cross the injector opening. Due to the spatial constraints of the experimental article, only a single flushwall injector could be installed and imaged. However, as before, the CFD and the corresponding CFI have been obtained for a row of injectors. Nevertheless, a direct comparison can still be made between PLIF and CFI as long as the compressible features (i.e., 

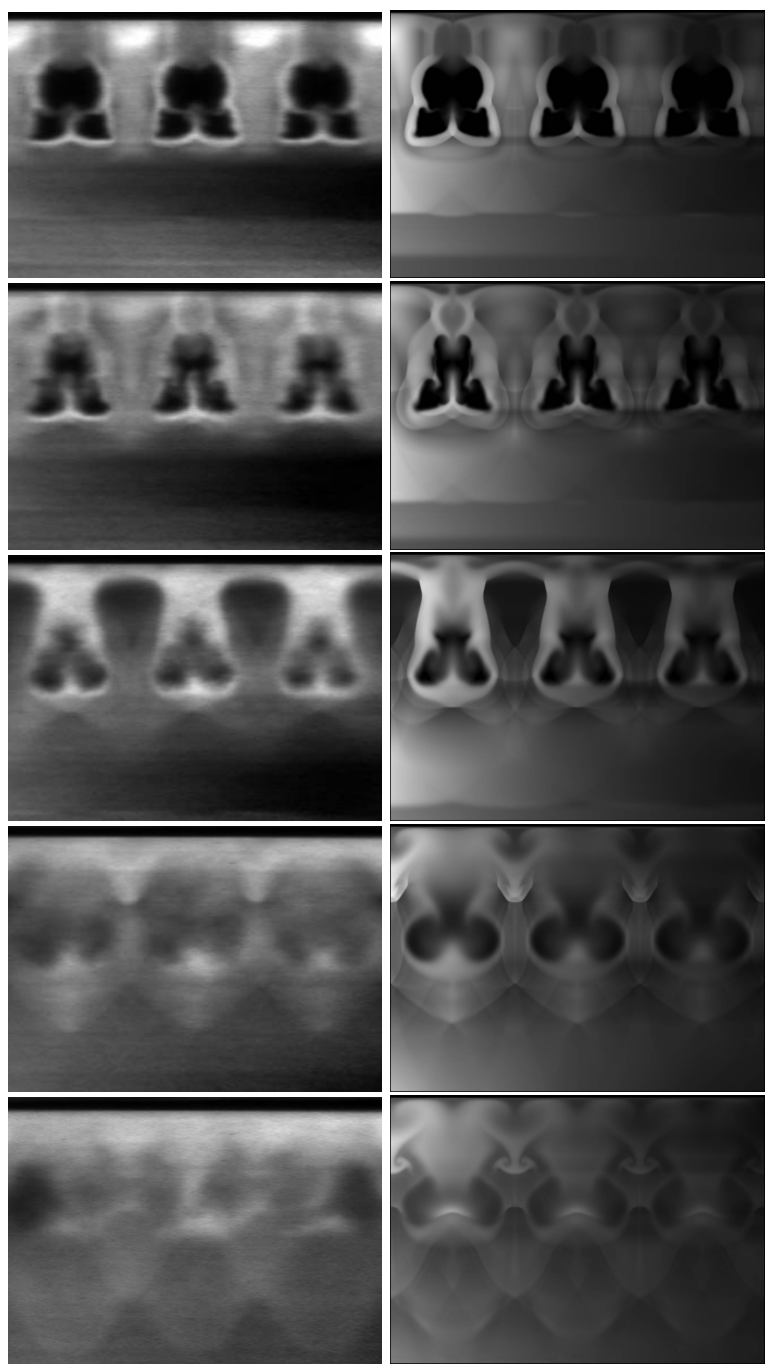

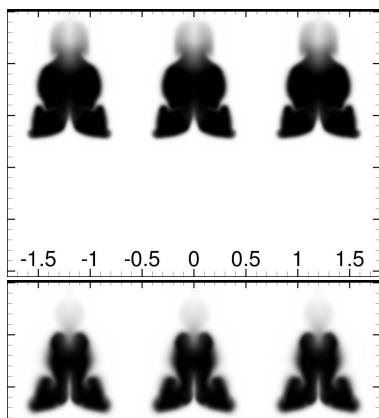

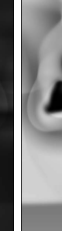
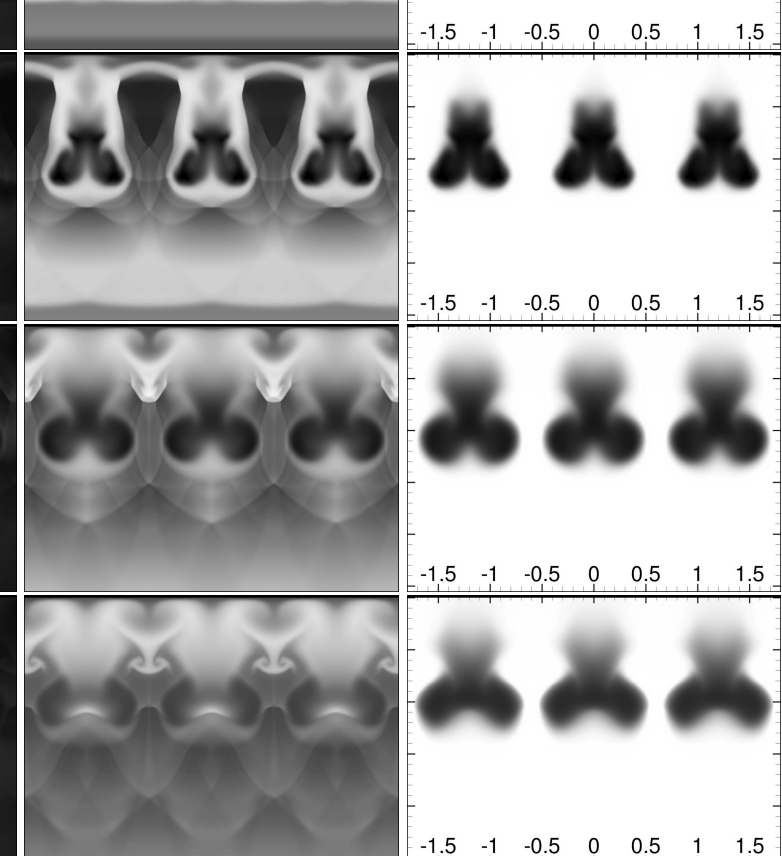

Figure 19. Time-averaged cross-stream PLIF images (left-most column) for the ramp injector, the corresponding CFI (two middle columns), and mole fraction of air (right-most column) at $0.5,1.0,2.0,4.0$, and 6.0 inches (top-to-bottom) downstream from the injector face. The two middle CFI are obtained using the mole fraction sensitive LIF transition lines, with the left set of images corresponding to LIF where Beer-Lambert absorption model was applied to the CFI on the right. The dimensions are in inches.

shocks and expansions) of neighboring injectors do not strongly interact. This is the case for all but the last two rows of images. Furthermore, the flushwall injector in the CFD is shifted about 1.49 inches downstream with respect to its experimental location. This shift is also a result of spatial restrictions on the experimental article that were unforeseen during the pretest CFD campaign. The only consequence of this shift, however, is that the bow shock emanating from the leading edge of the experimental plate appears closer to the plate (top of each image) in the PLIF images than the CFI. The boundary layer thickness does not significantly change over that shift distance, therefore, is not expected to significantly impact the results either. The PLIF and the CFI are obtained using the mole fraction sensitive transition lines. As above, a simple absorption model is used for the images in the left of the two central columns. This model does reproduce the characteristics absorption "shadows" visible to the right of the growing shock layer in the PLIF images. The CFI without this absorption model are shown on the right of the two central image columns. The qualitative agreement between the PLIF and CFI is similar to that observed above. However, one notable difference is the attenuation (darkening) of the CFI signal as the flow approaches the wall in the boundary layer. This attenuation is missing in all of the PLIF images. The cause of this effect is the difference in the flat plate temperature between the experiment and the CFD. In the experiments, the plate temperature using thermocouples reaches about $340 \mathrm{~K}$, 
whereas in the pretest CFD it was modeled using an adiabatic wall assumption resulting in the values of about $900 \mathrm{~K}$. Because the fluorescence signal is inversely proportional to the temperature, while Boltzmann fraction increases with the temperature for low temperatures then decreases, there exists a temperature for which the signal peaks then decays as the temperature continues to increase. For the mole fraction lines, the CFI equation reveals that the temperature for which the signal peaks is about $300 \mathrm{~K}$, which is consistent with PLIF images of the boundary layer.

For the flushwall injector the entrainment of air into the core of the fuel plume appears greater in the CFI than PLIF as evidenced by the larger extent of the signal inside the fuel plume. It also appears that the shock interactions with the neighboring injectors cause the fuel plume to stretch downward, thereby increasing the fuel penetration slightly.

\section{Summary and Conclusions}

The current work compares the experimentally-obtained and CFD-computed NO PLIF images of the mixing flowfields for three high-speed fuel injectors, with helium used as an inert substitute for hydrogen fuel. The three injectors consist of a strut, a ramp, and a high aspect ratio rectangular flushwall injector. These devices accomplish the task of distributing and mixing fuel into the supersonic crossflow, albeit via different strategies. The PLIF is obtained by utilizing NO naturally occurring in the Arc-Heated Scramjet Test Facility used for the experiments conducted at NASA Langley. Because the NO is present in the facility air, the absence of signal is an indication of pure fuel. Three different sets of fluorescence transition lines are used in the current work. Each set of lines exhibits different sensitivity to pressure, temperature, and mole fraction of NO, and has different absorption characteristics. Two of the transition lines have been selected for their linear correlation with either mass fraction or mole fraction of helium. The computed PLIF images are obtained by combining a weak excitation fluorescence model for NO with the ReynoldsAveraged Simulations carried out using the VULCAN-CFD solver to obtain a computational equivalent of the PLIF signal. This process produces computational flow imaging (CFI). The measured PLIF signal is mainly a function of $\mathrm{NO}$ concentration allowing for a semiquantitative comparisons between the mixing flowfields obtained from the CFI and experiments. The PLIF signal intensity is also sensitive to pressure and temperature variations in the flow, allowing additional flow features to be identified and compared with the CFI. The semiquantitative nature of the comparisons stems from the uncertainties in the instantaneous amount of background NO, the absorption modeling, the PLIF signal postprocessing, as well as uncertainties in the PLIF signal modeling itself. The laser energy absorption in the CFI is qualitatively modeled using a simple model motivated by the Beer-Lambert law. Reasonable agreement is observed between the experimental PLIF images and the CFI establishing an increased confidence in the postprocessing and modeling approaches, and the CFD simulations.

\section{Acknowledgments}

This work is supported by the Hypersonic Technology Project in the NASA Aeronautics Research Mission Directorate (ARMD). Experimental support was provided by the Supersonic and Hypersonic Testing Branch at the NASA Langley Research Center. Computational resources are provided by the NASA Langley Research Center and the NASA Advanced Supercomputing (NAS) Division.

\section{References}

\footnotetext{
$\rightarrow$ Peebles, C., Road to Mach 10: Lessons Learned From the X-43A Flight Research Program, Library of Flight Series, AIAA, Washington, D.C., 2008.

${ }^{2}$ Hank, J.M., Murphy, J.S., and Mutzman, R.C., "The X-51A Scramjet Engine Flight Demonstration Program," in 15th AIAA International Space Planes and Hypersonic Systems and Technologies Conference, AIAA, Dayton, OH, 2008.

${ }^{3}$ Lee, J., Lin, K.C., and Eklund, D., "Challenges in Fuel Injection for High-Speed Propulsion Systems," AIAA J., Vol. 53, No. 6, 2015, pp. $1405-1423$.

${ }^{4}$ Cabell, K., Drozda, T.G., Axdahl, E.L., and Danehy, P.M., "The Enhanced Injection and Mixing Project at NASA Langley," in JANNAF 46th CS / 34th APS / 34th EPSS / 28th PSHS Joint Subcommittee Meeting, Albuquerque, NM, 2014.

${ }^{5}$ Baurle, R.A., Fuller, R.P., White, J.A., Chen, T.H., Gruber, M.R., and Nejad, A.S., "An Investigation of Advanced Fuel Injection Schemes for Scramjet Combustion," in 36th Aerospace Sciences Meeting and Exhibit, Reno, NV, 1998.

${ }^{6}$ Ogawa, H., "Physical Insight into Fuel-Air Mixing for Upstream-Fuel-Injected Scramjets via Multi-Objective Design Optimization," $J$ Propul Power, Vol. 31, No. 6, 2015, pp. 1505-1523.

${ }^{7}$ Drozda, T.G., Drummond, J.P., and Baurle, R.A., "CFD Analysis of Mixing Characteristics of Several Fuel Injectors at Hypervelocity Flow Conditions," in 52nd AIAA/SAE/ASEE Joint Propulsion Conference, AIAA 2016-4764, AIAA, Salt Lake City, UT, 2016.
} 


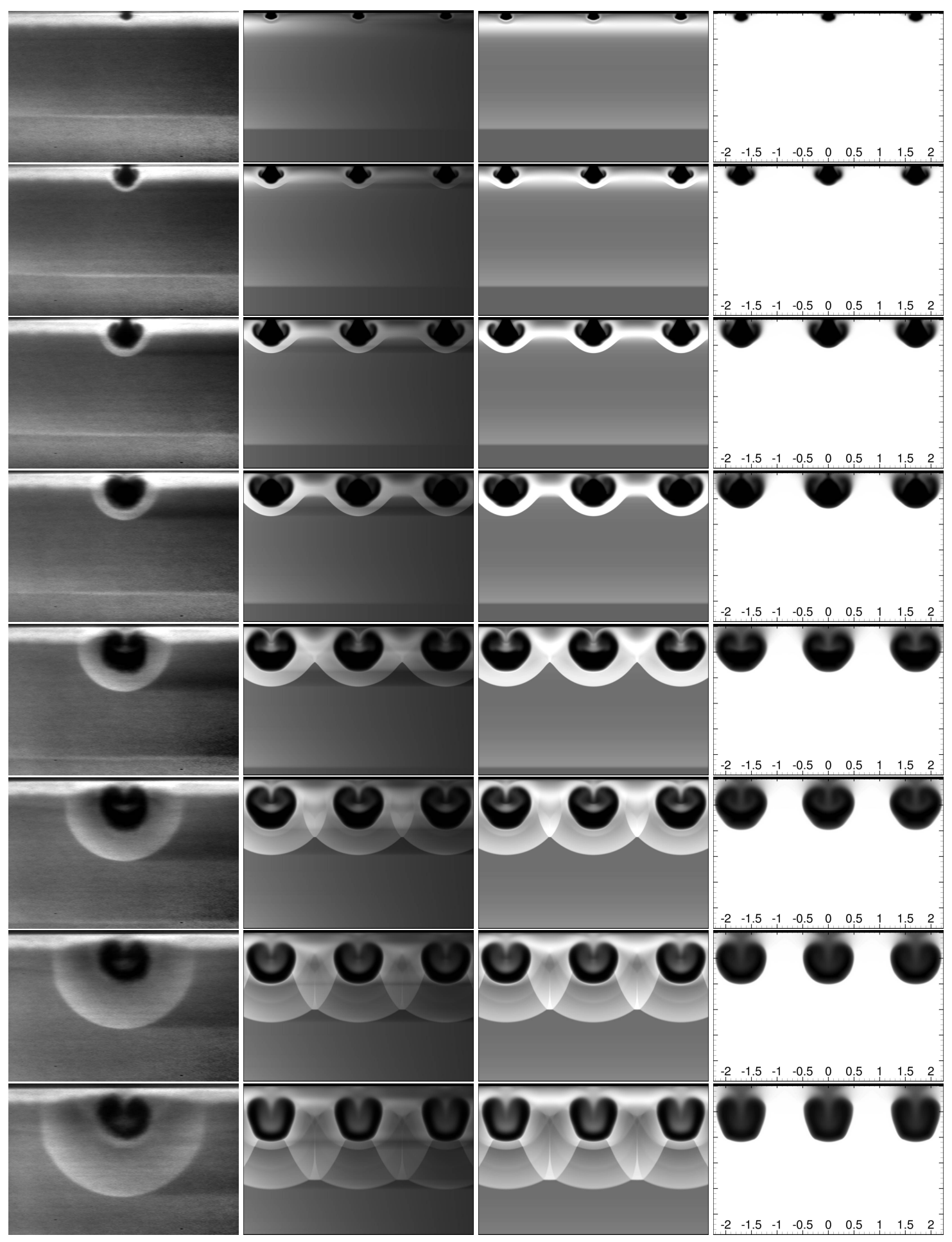

Figure 20. Time-averaged cross-stream PLIF images (left-most column) for the flushwall injector, the corresponding CFI (two middle

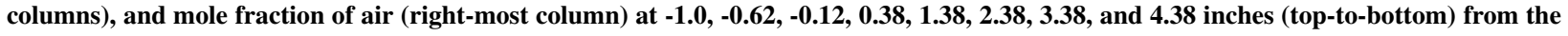
injector trailing edge. The two middle CFI are obtained using the mole fraction sensitive LIF transition lines, with the left set of images corresponding to LIF where Beer-Lambert absorption model was applied to the CFI on the right. The experimental PLIF images contain only one injector whereas the CFD modeled a row of injectors. The dimensions are in inches. 
${ }^{8}$ Cabell, K.F. and Rock, K.E., "A Finite Rate Chemical Analysis of Nitric Oxide Flow Contamination Effects on Scramjet Performance," Tech. Rep. TP-212159, NASA, 2003.

${ }^{9}$ Hartfield, R.J., Abbitt, J., , and McDaniel, J.C., "Injectant Mole-Fraction Imaging in Compressible Mixing Flows Using Planar LaserInduced Iodine Fluorescence," Opt. Lett., Vol. 16, No. 16, 1989, pp. 850-852.

${ }^{10}$ Clemens, N.T. and Mungal, M.G., "Large-Scale Structure and Entrainment in the Supersonic Mixing Layer," J. Fluid Mech., Vol. 284, 1995, pp. 171-216.

${ }^{11}$ Island, T.C., Urban, W.D., and Mungal, M.G., "Quantitative Scalar Measurements in Compressible Mixing Layers," in 34th AIAA Aerospace Sciences Meeting and Exhibit, AIAA, AIAA, Reno, NV, 1996.

${ }^{12}$ Donohue, J.M. and McDaniel, J.C., "Computer-Controlled Multiparameter Flowfield Measurements Using Planar Laser-Induced Iodine Fluorescence," AIAA J., Vol. 34, No. 8, 1996, pp. 1604-1611.

${ }^{13}$ Fox, J.S., Houwing, A.F.P., Danehy, P.M., Gaston, M.J., Mudford, N.R., and Gai, S.L., "Mole-Fraction-Sensitive Imaging of Hypermixing Shear Layers," J. Propul. Power., Vol. 17, No. 2, 2001, pp. 284-292.

${ }^{14}$ Gaston, M.J., Houwing, A.F.P., Mudford, N.R., Danehy, P.M., and Fox, J.S., "Fluorescence Imaging of Mixing Flowfields and Comparisons with Computational Fluid Dynamic Simulations," Shock Waves, Vol. 12, 2002, pp. 99-110.

${ }^{15}$ Rossmann, T., Mungal, M.G., and Hanson, R.K., "Nitric-Oxide Planar Laser-Induced Fluorescence Applied to Low-Pressure Hypersonic Flow Fields for the Imaging of Mixture Fraction," Appl. Opt., Vol. 42, No. 33, 2003, pp. 6682-6695.

${ }^{16}$ Rossmann, T., Mungal, M.G., and Hanson, R.K., "Mixing Efficiency Measurements Using a Modified Cold Chemistry Technique," Exp. Fluids, Vol. 37, No. 4, 2004, pp. 566-576.

${ }^{17}$ Takahashi, H., Ikegami, S., Oso, H., Masuya, G., and Hirota, M., "Quantitative Imaging of Injectant Mole Fraction and Density in a Supersonic Mixing," AIAA J., Vol. 46, No. 11, 2008, pp. 2935-2943.

$\checkmark{ }^{18}$ Kidd III, F.G., Narayanaswamy, V., Danehy, P.M., Inman, J.A., Bathel, B.F., Cabell, K.F., Hass, N.E., Capriotti, D.P., Drozda, T.G., and Johansen, C.T., "Characterization of the NASA Langley Arc Heated Scramjet Test Facility Using NO PLIF," in 30th AIAA Aerodynamic Measurement Technology and Ground Testing Conference, Atlanta, GA, 2014.

${ }^{19}$ Ivey, C.B., Danehy, P.M., Bathel, B.F., Dyakonov, A.A., Inman, J.A., and Jones, S.B., "Comparison of PLIF and CFD Results for the Orion CEV RCS Jets," in 49th AIAA Aerospace Sciences Meeting, AIAA-2011-713, AIAA, Orlando, FL, 2011.

${ }^{20}$ VULCAN-CFD, "http://vulcan-cfd.larc.nasa.gov/," 2016.

${ }^{21}$ Drozda, T.G., Axdahl, E.L., and Cabell, K.F., "Pre-Test CFD for the Design and Execution of the Enhanced Injection and Mixing Project at NASA Langley Research Center," in JANNAF 46th CS / 34th APS / 34th EPSS / 28th PSHS Joint Subcommittee Meeting, Albuquerque, NM, 2014.

22 Drozda, T.G., Cabell, K.F., Passe, B.J., and Baurle, R.A., "Computational and Experimental Characterization of the Mach 6 Facility Nozzle Flow for the Enhanced Injection and Mixing Project at NASA Langley Research Center," in AIAA SciTech Forum, AIAA, Grapevine, TX, 2017.

${ }^{23}$ Brown, G.L. and Roshko, A., "On Density Effects and Large Structure in Turbulent Mixing Layers," J. Fluid Mech., Vol. 64, 1974, pp. 775-816.

24 Bogdanoff, D.W., "Compressibility Effects in Turbulent Shear Layers," AIAA J., Vol. 21, No. 6, 1983, pp. 926-927.

25 Papamoschou, D. and Roshko, A., "The Compressible Turbulent Shear Layer: An Experimental Study," J. Fluid Mech., Vol. 197, 1988, pp. 453-477.

${ }^{26}$ Gruber, M.R., Nejad, A.S., and Dutton, J.C., "Circular and Elliptical Transverse Injection into a Supersonic Crossflow - The Role of Large-Scale Structures," in 26th Fluid Dynamics Conference, San Diego, CA, 1995.

${ }^{27}$ Schetz, J.A. and Billig, F.S., "Penetration of Gaseous Jets Injected into a Supersonic Stream," J Spacecraft Rockets, Vol. 3, No. 11, 1966, pp. $1658-1665$.

${ }^{28}$ Mao, M., Riggins, D.W., and McClinton, C.R., "Numerical Simulation of Transverse Fuel Injection,” in Computational Fluid Dynamics Symposium on Aeropropulsion, NASA-CP-3078, NASA, Cleveland, OH, 1990, pp. 635-667.

$\checkmark 29$ Portz, R. and Segal, C., "Penetration of Gaseous Jets in Supersonic Flows," AIAA J, Vol. 44, No. 10, 2006, pp. $2426-2429$.

${ }^{30}$ McClinton, C.R., "Effect of Ratio of Wall Boundary-Layer Thickness to Jet Diameter on Mixing of a Normal Hydrogen Jet in Supersonic Stream," NASA Technical Memorandum NASA TM X-3030, NASA, Hampton, VA, 1974.

${ }^{31}$ van Leer, B., "Towards the Ultimate Conservative Difference Scheme. V: A Second-Order Sequel to Godunov's Method," J. Comput. Phys., Vol. 32, No. 1, 1979, pp. 101-136.

-32Edwards, J.R., "A Low-Diffusion Flux-Splitting Scheme for Navier-Stokes Calculations," Comput. Fluids., Vol. 26, No. 6, 1997, pp. 635659 .

${ }^{33}$ McBride, B.J., Gordon, S., and Reno, M.A., "Thermodynamic Data for Fifty Reference Elements," NASA Technical Paper 3287/REV1, NASA, Cleveland, OH, 2001.

${ }^{34}$ Pulliam, T.H. and Chaussee, D.S., "A Diagonal Form of an Implicit Approximate-Factorization Algorithm,” J. Comput. Phys., Vol. 39, No. 2, 1981, pp. 347-363.

${ }^{35}$ Menter, F.R., "Two-Equation Eddy-Viscosity Turbulence Models for Engineering Applications," AIAA J., Vol. 32, No. 8, 1994, pp. 15981605 .

${ }^{36}$ Wilcox, D.C., Turbulence Modeling for CFD, DCW Industries, Inc., La Cañada, CA, 2000.

- ${ }^{37}$ Waitz, I., Marble, F., and Zukoski, E., "Vorticity Generation by Contoured Wall Injectors," in AIAA/SAE/ASME/ASEE 28th Joint Propulsion Conference and Exhibit, AlAA 92-3550, AIAA, Nashville, TN, 1992.

${ }^{38}$ Palma, P.C., Laser-Induced Fluorescence Imaging in Free-Piston Shock Tunnels, Phd dissertation, Australian National University, 1999.

${ }^{39}$ Schneider, C.A., Rasband, W.S., and Eliceiri, K.W., "NIH Image to ImageJ: 25 Years of Image Analysis," Nat. Methods, Vol. 9, No. 7, 2012, pp. 671-675.

${ }^{40}$ Paul, P.H., Gray, J.A., Durant Jr., J.L., and Thoman Jr., J.W., "Collisional Quenching Corrections for Laser-Induced Fluorescence Measurements of NO $A^{2} \Sigma^{+}, " A I A A J .$, Vol. 32, No. 8, 1994, pp. 1670-1675.

${ }^{41}$ Eckbreth, A.C., "Laser Diagnostics for Combustion Temperature and Species," 1996. 
${ }^{42}$ Danehy, P.M., Palma, P.C., Boyce, R.R., and Houwing, A.F.P., "Numerical Simulations of Laser-Induced Fluorescence Imaging in ShockLayer Flows," AIAA J., Vol. 37, 1999, pp. 715-722. 